\title{
Variational integrators for reduced field equations
}

\author{
Ana Casimiro $^{1, *}$, César Rodrigo ${ }^{2}$ \\ ${ }^{1}$ CMA - Centro de Matemática e Aplicações, Departamento de Matemática, Faculdade de Ciências e Tecnologia, \\ Universidade Nova de Lisboa, Quinta da Torre 2829-516 Caparica, Portugal \\ ${ }^{2}$ CMAF-CIO - Centro de Matemática, Aplicações Fundamentais e Investigação Operacional, \\ CINAMIL, Academia Militar, Av. Conde Castro Guimarães, 2720-113 Amadora, Portugal
}

\begin{abstract}
In the reduction of field theories in principal $G$-bundles, when a subgroup $H \subset G$ acts by symmetries of the Lagrangian, each of the $H$-reduced unknown fields decomposes as a flat principal connection and a parallel $\mathrm{H}$-structure. A suitable variational principle with differential constraints on such fields leads to necessary criticality conditions known as Euler-Poincaré equations. We model constrained discrete variational theories on a simplicial complex and generate from the smooth theory, in a covariant way, a discrete variational formulation of $H$-reduced field theories. Critical fields in this formulation are characterized by a corresponding discrete version of Euler-Poincaré equations. We present a numerical integration algorithm for discrete Euler-Poincaré equations, that extends integration algorithms for Euler-Poincaré equations in mechanics to the case of field theories and, also, extends integration algorithms for Euler-Lagrange equations in discrete field theories to the case of variational principles with constraints. For regular reduced discrete Lagrangians, this algorithm allows to univocally propagate initial condition data, on an initial condition band, into a solution of the corresponding equations for the discrete variational principle.
\end{abstract}

Keywords Variational Integrator, Reduction, Euler-Poincaré equation, Simplicial complex, Discretization

AMS 2010 subject classifications 39A12, 49M25, 37J15, 65D25, 65D30

DOI: $10.19139 /$ soic.v6i1.469

\section{Introduction}

In the community working on numerical methods for ordinary and partial differential equations arising in physics, it has become clear in the last years that discretising the variational principles, that originally generated the equations, is a very fruitful method, when we are looking for some discrete version of the equations and integrators for this discrete version. In such a way that, the usual geometrical considerations in the smooth theory also make sense for the discretized versions of the equations and for its solutions. Following this guideline one usually is rewarded with integrators with simplecticity and discrete conservation laws.

In this line of ideas a large variety of situations have been studied, with a relevant focus in all variants of mechanics (with or without constraints, control theory, reduced theories) [11, 12, 19, 24, 27, 28, 30, 35, 42]. Fewer papers explore the challenging case of field theories [14, 20, 21, 22, 31, 32, 33, 40].

It is in the area of field theory that many aspects on the discretization of variational principles remain unsolved. A common approach is to treat the discrete field theory with large-dimensional functional spaces, like in the theory of finite elements [1]. In many other situations the corresponding field theory has a natural time variable and its discretization allows for a treatment similar to that of discrete mechanical systems $[14,15]$. These examples

\footnotetext{
*Correspondence to: Ana Casimiro (Email: amc@fct.unl.pt). Departamento de Matemática, Faculdade de Ciências e Tecnologia,
} Universidade Nova de Lisboa, Quinta da Torre 2829-516 Caparica, Portugal.

ISSN 2310-5070 (online) ISSN 2311-004X (print)

Copyright (C) 2018 International Academic Press 
illustrate that, from a geometrical point of view, and except for some few cases, the discretization of field theory is performed with very little concern about the nature of the underlying discrete manifold of independent variables. In fact, most examples take as discrete independent variables some bidimensional cartesian grid.

The reduction of mechanical systems on Lie groups, where critical trajectories are characterized by EulerPoincaré equations, has also been treated in the literature in $[2,25,26,34]$ and its extension to field theories has been explored [7, 40], but with several simplifying restrictions, as is the consideration of trivial principal bundles, and of a cartesian bidimensional grid as base space.

Going one step further, in the smooth theory of gauge fields the reduction process leads to the consideration of bundles of connections. The discrete analogue of a connection has been described by several authors $[13,17,29,36,38,41]$, mostly due to concerns of its applications to mechanical systems, and consequently, for the case of a Lie group. Much more remains to be said about discrete connections on a principal bundle.

In a recent work by the authors [6], we have described a procedure to discretize the key element of a variational principle: its Lagrangian density. This work shows how the discretization mechanism can be performed in such a way that it commutes with a possible reduction, by the action of some group of symmetries $H$. This leads to get a covariant way to define discrete $H$-reduced Lagrangian densities, it remains to explore how such an object generates a variational principle that represents the discrete analogue of Euler-Poincaré reduction, developed in the smooth case in [6].

In the present work we show how, starting from a discrete $H$-reduced Lagrangian density, one gets a discrete variational principle for $H$-reduced fields. This principle retains the structure of the smooth one $[8,9,10,16]$. Fields are discrete $H$-structure together with a discrete connection. There is a constraint on the curvature and another to ensure the $H$-structure is parallel. Admissible variations are restricted to a subset given by infinitesimal gauge transformations. Finally critical fields for the variational principle are characterized by a set discrete Euler-Poincaré equations. We study the structure of these equations and identify the relevant momentum mapping for this situation. As happened in non-reduced discrete field theories [5], the existence of an inverse of the momentum mapping (what we call an integrator) is the central element in an iterative algorithm, that allows to extend admissible $H$-reduced fields on some initial band to globally defined fields, that are critical for the discrete Euler-Poincaré variational principle.

The work begins in section 2 with the presentation of the main tools of Euler-Poincaré reduction, including an introduction to the variational principles that lead to smooth Euler-Poincaré equations, with the relevant Theorem 2.2 that relates the original and reduced variational principles. This section also includes fundamental results obtained in [6], related to our problem. Next section 3 presents our discretization choice for the space, an abstract simplicial complex arising from the Coxeter-Freudenthal-Kuhn simplicial partition of a cartesian lattice. Section 4 introduces variational principles on discrete bundles of $H$-structures and principal connections. In Proposition 4.4 the differential of the action functional is represented in a trivialized version, expressing it in terms of linear functions on the adjoint bundle. Section 5 introduces then the discrete version of the Euler-Poincaré constrained variational principle. Critical points for this principle are then characterized in Theorem 5.1, using a discrete version of the Euler-Poincaré equations. The particular structure of these equations is explored in section 6 . The Legendre and momentum mappings are identified as the relevant components in the equations, and we show that the possibility to integrate the equations lies in the existence of an inverse of the Legendre mapping. If we assume that the Lagrangian density has an inverse operator for the Legendre mapping, such an operator is called an integrator and we describe the algorithm that generates solutions from any admissible initial condition band.

Certain technical aspects related to quotient manifolds and polytopes are included in the Appendix section.

\section{Reduction to Euler-Poincaré equations}

\subsection{Euler-Lagrange equations for fields on a fibered manifold}

We recall here the fundamentals of the calculus of variations on fibred manifolds. Let $\pi: Y \rightarrow X$ be a smooth bundle. Using local coordinates $\left(x^{1}, \ldots, x^{n}\right)$ on the base manifold $X$, we get corresponding functions $x^{i} \circ \pi$ on the bundle $Y$, that we denote by $\left(x^{1}, \ldots, x^{n}\right)$ and can be locally completed to a system of coordinate functions 
$\left(x^{1}, \ldots, x^{n}, y^{1}, \ldots, y^{m}\right)$ on $Y$. Mappings $y: U \rightarrow Y$ defined on open subsets $U \subseteq X$ and such that $\pi \circ y=\operatorname{Id}_{U}$ (local sections of the bundle) are called local fields and can be expressed in such a system of local coordinates as a family of smooth functions $y^{i}\left(x^{1}, \ldots, x^{n}\right)$. We denote by $\Gamma(Y)$ the set of local sections and by $\Gamma(K, Y)$ the subset of local sections whose domain contains $K \subseteq X$.

Consider the first jet bundle $J Y$ of sections, associated to the bundle $Y$, a bundle with local coordinates $\left(x^{\nu}, y^{i}, y_{\nu}^{i}\right)$. Local sections $y \in \Gamma(Y)$ of $\pi: Y \rightarrow X$ determined by equations $y^{i}=y^{i}\left(x^{1}, \ldots, x^{n}\right)$ induce a corresponding jet extension $j^{1} y \in \Gamma(J Y)$, a local section of $j \pi: J Y \rightarrow X$ determined by equations $y^{i}=$ $y^{i}\left(x^{1}, \ldots, x^{n}\right), y_{\nu}^{i}=\left(\partial y^{i} / \partial x^{\nu}\right)\left(x^{1}, \ldots, x^{n}\right)$.

Consider the vertical bundle $\pi_{V Y}: V^{\pi} Y \rightarrow Y$ determined by all tangent vectors $\delta_{y} \in T Y$ that are vertical for the projection $\mathrm{d} \pi: T Y \rightarrow T X$ (when the projector under consideration is clear, we shall simply write $V Y$ instead of $\left.V^{\pi} Y\right)$. This is a vector bundle on $Y$ and any system of fibred coordinates $\left(x^{1}, \ldots, x^{n}, y^{1}, \ldots, y^{m}\right)$ induces linear coordinates $\alpha^{i}$, so that each element $\delta_{y} \in V Y$ can be written as $\delta_{y}=\alpha^{i}\left(\partial / \partial y^{i}\right)_{y}$ (we use Einstein's convention for summation on repeated indices). In a similar way as local sections have a natural extension to the jet bundle, also local sections of $\pi \circ \pi_{V Y}: V Y \rightarrow X$ have a natural extension to $V(J Y)$. For any given vertical vector field $\delta_{y} \in \Gamma(V Y)$ defined on a local section $y=\pi_{V Y} \circ \delta_{y} \in \Gamma(Y)$, with local expression $\alpha^{i}=\alpha^{i}\left(x^{1}, \ldots, x^{n}\right)$ and $y^{i}=y^{i}\left(x^{1}, \ldots, x^{n}\right)$, there is a naturally induced local section $D_{j^{1} y}=j^{1} \delta_{y} \in \Gamma(V(J Y))$, projecting to $\delta_{y}$ when we use $\mathrm{d} \pi_{J}: V(J Y) \rightarrow V Y$, projecting to $j^{1} y \in \Gamma(J Y)$ when we use $\pi_{V(J Y)}: V(J Y) \rightarrow J Y$, and locally given as $D_{j^{1} y}=\alpha^{i}\left(\partial / \partial y^{i}\right)_{j^{1} y}+\beta_{\nu}^{i}\left(\partial / \partial y_{\nu}^{i}\right)_{j^{1} y}$ with:

$$
\beta_{\nu}^{i}=\left(\partial \alpha^{i} / \partial x^{\nu}\right)\left(x^{1}, \ldots, x^{n}\right)
$$

this section $D_{j^{1} y}=j^{1} \delta_{y}$ is called the vertical infinitesimal contact transformation associated to $\delta_{y} \in \Gamma(V Y)$.

We should recall that $\pi \circ \pi_{V Y}: V Y \rightarrow X$ is not a vector bundle. However for any fixed section $y \in \Gamma(Y)$, we have a vector bundle $y^{*} V Y \rightarrow X$ defined as the pull-back (or restriction) of the bundle $V Y \rightarrow Y$ along the local section $y: U \rightarrow Y$. Observe that a smooth variation of $y_{0} \in \Gamma(Y)$, that is, a smooth mapping $y_{\epsilon}\left(x^{1}, \ldots, x^{n}\right):\left[0, \epsilon_{\max }\right] \times U \rightarrow Y$ where $y_{\epsilon} \in \Gamma(Y)$ have a common domain $U \subset X$, allows to determine the associated infinitesimal variation $\delta_{y_{0}}=(d / d \epsilon)_{0}\left(y_{\epsilon}\left(x^{1}, \ldots, x^{n}\right)\right) \in \Gamma\left(y_{0}^{*} V Y\right)$, and its associated vertical infinitesimal contact transformation fulfills:

$$
j^{1} \delta_{y_{0}}=(d / d \epsilon)_{0}\left(\left(j^{1} y_{\epsilon}\right)\left(x^{1}, \ldots, x^{n}\right)\right) \in \Gamma\left(\left(j^{1} y_{0}\right)^{*} V(J Y)\right)
$$

Variational problems on a bundle $\pi: Y \rightarrow X$ arise when we fix a smooth function $\mathcal{L}$ on $J Y$ (the Lagrangian) and a smooth volume form $\operatorname{vol}_{X} \in \Omega^{n}(X)$ on $X$. The product $\mathcal{L} \operatorname{vol}_{X}$ is a horizontal $n$-form on $J Y$, and determines an action functional $\mathbb{L}$, a real-valued mapping that associates to any compact domain $K \subset X$ and local field $y \in \Gamma(K, Y)$, defined on an open neighbourhood of the domain, a value called the action associated to the field on the domain $K$ :

$$
\mathbb{L}_{K}(y)=\int_{K}\left(\mathcal{L} \circ j^{1} y\right) \operatorname{vol}_{X}
$$

Variational problems try to characterize critical points for the action functional, with respect to certain admissible variations. Indeed, if $\left(y_{\epsilon}\right)_{0 \leq \epsilon \leq \epsilon_{\max }} \subset \Gamma(K, Y)$ is a smooth variation of a section $y_{0} \in \Gamma(K, Y)$ for some compact domain $K \subset X$, derivating inside of the integral symbol and using the chain rule together with (1) we have the expression for the first derivative of $\mathbb{L}_{K}\left(y_{\epsilon}\right)$ :

$$
\left(\frac{\mathrm{d}}{\mathrm{d} \epsilon}\right)_{0} \mathbb{L}_{K}\left(y_{\epsilon}\right)=\int_{K}\left\langle\mathrm{~d}_{y_{0}}^{v} \mathcal{L}, j^{1} \delta_{y_{0}}\right\rangle \operatorname{vol}_{X}, \quad \delta_{y_{0}}=\left(\frac{\mathrm{d}}{\mathrm{d} \epsilon}\right)_{0} y_{\epsilon} \in \Gamma\left(y_{0}^{*} V Y\right)
$$

where $\mathrm{d} \mathcal{L} \in \Gamma\left(T^{*}(J Y)\right)$ determines $\mathrm{d}_{y_{0}}^{v} \mathcal{L} \in \Gamma\left(\left(j^{1} y_{0}\right)^{*} V^{*}(J Y)\right)$ using the restriction to the section $j^{1} y_{0}$ and the restriction to $V(J Y) \subset T(J Y)$, and where $\langle\cdot, \cdot\rangle$ represents the duality product with elements in $\Gamma\left(\left(j^{1} y_{0}\right)^{*} V(J Y)\right)$.

The integration $\int_{K}\left(j^{1} y\right)^{*} \mathcal{L} \operatorname{vol}_{X}$ in the action functional is a real value if we assume that we are working with a compact domain $K \subseteq X$ (for example we may assume $K=X$ in the case that $X$ is compact). If we do not fix a compact domain $K \subset X$, we do not have a well defined value $\mathbb{L}(y)$ for every $y \in \Gamma(Y)$. However, the linear functional $\mathrm{d}_{y} \mathbb{L}: \delta_{y} \in \Gamma\left(y^{*} V Y\right) \mapsto \int_{X}\left\langle\mathrm{~d}_{y_{0}}^{v} \mathcal{L}, j^{1} \delta_{y}\right\rangle \operatorname{vol}_{X} \in \mathbb{R}$ makes sense with the simple condition that 
$\delta_{y} \in \Gamma\left(y^{*} V Y\right)$ is chosen with a support that is compact in the domain of $y \in \Gamma(Y)$, no matter that this domain might be a non-compact subset $U \subset X$.

Definition 2.1

For any local section $y: U \rightarrow Y$ of the bundle $\pi: Y \rightarrow X$, we call differential at $y$ of the action functional $\mathbb{L}$, associated to a Lagrangian density $\mathcal{L}_{\mathrm{vol}_{X}}$, the linear functional:

$$
\mathrm{d}_{y} \mathbb{L}: \delta_{y} \in \Gamma^{c}\left(y^{*} V Y\right) \mapsto \int_{U}\left\langle\mathrm{~d}_{y_{0}}^{v} \mathcal{L}, j^{1} \delta_{y}\right\rangle \operatorname{vol}_{X} \in \mathbb{R}
$$

where the subspace $\Gamma^{c}\left(y^{*} V Y\right)$ is given by all sections of the bundle $y^{*} V Y \rightarrow U$ whose support is a compact subset $K \subset U$.

A general formulation of a variational problem with differential constraints on a fibred manifold $\pi: Y \rightarrow X$ (see [18]) tries to determine which local sections $y \in \Gamma(y)$ are critical, in the following sense:

\section{Definition 2.2}

Let $\pi: Y \rightarrow X$ be a bundle on an $n$-dimensional manifold, oriented by a volume element $\operatorname{vol}_{X} \in \Omega^{n}(X)$. A constrained variational problem is determined by:

- A smooth submanifold $\mathcal{S} \subseteq J Y$ (the constraint submanifold).

(A local field $y \in \Gamma(Y)$ is called admissible for the variational problem if $j^{1} y$ takes values on $\mathcal{S}$. The set of admissible local fields shall be denoted by $\Gamma_{\mathcal{S}}(Y)$.)

- Linear subspaces $\mathcal{A} \mathcal{V}_{y} \subset \Gamma^{c}\left(y^{*} V Y\right)$, for each admissible local field $y \in \Gamma_{\mathcal{S}}(Y)$, compatible with $\mathcal{S}$, that is, so that each $\delta_{y} \in \mathcal{A} \mathcal{V}_{y}$ determines a vertical contact transformation $j^{1} \delta_{y} \in \Gamma\left(\left(j^{1} y\right)^{*} V(J Y)\right)$ tangential to $\mathcal{S} \subseteq J Y$.

(Elements $\delta_{y} \in \mathcal{A} \mathcal{V}_{y} \subset \Gamma^{c}\left(y^{*} V Y\right)$ shall be called admissible infinitesimal variations at the admissible local field $y \in \Gamma_{\mathcal{S}}(Y)$.)

- A smooth function $\mathcal{L}: \mathcal{S} \rightarrow \mathbb{R}$.

(Determining a horizontal $n$-form $\mathcal{L}_{\mathrm{Vol}_{X}}$ called Lagrangian density, the corresponding action functional $\mathbb{L}_{K}: \Gamma_{\mathcal{S}}(Y) \rightarrow \mathbb{R}$ associated to any compact subset $K \subseteq X$, defined by (2) on the subset of admissible local fields, and the differential $\mathrm{d}_{y} \mathbb{L}: \mathcal{A} \mathcal{V}_{y} \rightarrow \mathbb{R}$ of the action functional, well defined by (3) on the subspace $\mathcal{A} \mathcal{V}_{y}$ of admissible infinitesimal variations $\mathcal{A} \mathcal{V}_{y}$ at any admissible local field $y \in \Gamma_{\mathcal{S}}(Y)$.)

An admissible local field $y \in \Gamma_{\mathcal{S}}(Y)$ is called critical for the variational problem if $\mathrm{d}_{y} \mathbb{L}$ vanishes on the space of admissible infinitesimal variations $\mathcal{A} \mathcal{V}_{y}$.

For the particular case of $\mathcal{S}=J Y$ and $\mathcal{A} \mathcal{V}_{y}=\Gamma^{c}\left(y^{*} V Y\right.$ ) (a situation that we denote as fixed boundary variations) criticality is equivalent to the annihilation of the Euler-Lagrange tensor $\mathcal{E} \mathcal{L}(y) \in \Gamma\left(y^{*} V^{*} Y\right)$, that for the case $\operatorname{vol}_{X}=\mathrm{d} x^{1} \wedge \ldots \wedge \mathrm{d} x^{n}$ takes the classical form:

$$
\mathcal{E} \mathcal{L}(y)=\left(\frac{\partial \mathcal{L}}{\partial y^{i}}\left(j_{x}^{1} y\right)-\frac{\mathrm{d}}{\mathrm{d} x^{\nu}}\left(\frac{\partial \mathcal{L}}{\partial y_{\nu}^{i}}\left(j_{x}^{1} y\right)\right)\right) \mathrm{d} y^{i} \in \Gamma\left(y^{*} V^{*} Y\right)
$$

the appearance of $\left(\mathrm{d} / \mathrm{d} x^{\nu}\right)$ in an object depending on $j^{1} y$ shows that equations $\mathcal{E} \mathcal{L}(y)=0$ represents a system of second order partial differential equations on the unknown $y \in \Gamma(Y)$.

For a different choice of admissible infinitesimal variations, however, critical sections can be characterized by a different system of partial differential equations. This is, for example, the case of Euler-Poincare equations in the case of gauge field theories invariant by the action of a certain group $H$. These equations arise as necessary criticality conditions for a variational problem with constraints that we introduce next.

\subsection{H-structures on a principal G-bundle}

An action (also called action on the left) of a group $G$ on a set $M$ is a group morphism $\lambda: g \in G \rightarrow \lambda_{g} \in \operatorname{Aut}(M)$ $\left(\lambda_{g h}=\lambda_{g} \circ \lambda_{h}, \lambda_{e}=\mathrm{Id}_{M}\right)$. We reserve the expression "left action" for the case $M=G$, to represent the specific 
mapping $l: h \in G \mapsto l_{h} \in \operatorname{Aut}(G)$ where $l_{h}(g)=h g$, and the expression "right action" for the specific mapping $r^{-1}: h \in G \mapsto r_{h}^{-1} \in \operatorname{Aut}(G)$ where $r_{h}^{-1}=g h^{-1}$. Both of them determine actions (on the left) of the group $G$ on the set $G$. In the case that $G, M$ are smooth manifolds, the group morphism $\lambda$ determines a mapping

$$
\left(a c, \pi_{M}\right):(g, m) \in G \times M \mapsto\left(\lambda_{g}(m), m\right) \in M \times M
$$

We say the action is smooth if $\left(a c, \pi_{M}\right)$ is smooth, say the action is free if $\left(a c, \pi_{M}\right)$ is injective and say the action is proper if $\left(a c, \pi_{M}\right)$ is proper (inverse image of compact sets are compact sets).

We will be concerned with Lie groups: groups with a smoth manifold structure, such that both the left and right actions $l, r^{-1}$ of $G$ on itself are smooth actions. For any Lie group $(G, \cdot)$ there exists a corresponding reverse Lie group $G^{r}=(G, \circ)$ using $G$ itself as manifold and the reverse product $g \circ h=h \cdot g$. We shall avoid talking of actions of $G$ on the right on $M$, that can be defined as actions of the reverse group $G^{r}$ on $M$. Smooth free proper actions of Lie groups on smooth manifolds will appear in this work and the existence of the quotient manifold for such actions is a classical result, with main properties expressed in the appendix. In particular, we shall introduce principal $G$-bundles with the action on the left convention:

\section{Definition 2.3}

Let $G$ be a Lie group. A principal $G$-bundle is a smooth manifold $P$, together with a free proper smooth action of a Lie group $G$ on $P$.

The fact that the smooth free action is proper warrants that there exists a unique quotient manifold structure $P / G$, such that the quotient mapping $\pi^{G}: P \rightarrow P / G$ is a surjective submersion. This submersion is usually called the fiber bundle structure, and the quotient manifold $X=P / G$ is called the base manifold of the principal bundle.

We should observe that for any given closed subgroup $H \subseteq G$, the same manifold $P$ has also a principal $H$ bundle structure considering the restriction of the action to $H \subseteq G$ (the mapping ( $\lambda$, Id) is still proper because $H$ is a closed subset). The quotient morphism $\pi^{H}: P \rightarrow P / H$ represents the fiber bundle structure of $P$ seen as a principal $H$-bundle. Fixing a principal $G$-bundle $\pi^{G}: P \rightarrow P / G=X$ and a closed subgroup $H \subseteq G$, we get a principal $H$-bundle $\pi^{H}: P \rightarrow H$ Str on the quotient manifold $H$ Str $=P / H$, the manifold of $H$-structures.

We observe that elements on the same $H$-orbit project to a unique point by $\pi^{G}: P \rightarrow X$, therefore $\pi^{G}$ factors by a morphism $\pi_{H S t r}: H \operatorname{Str}=P / H \rightarrow X$. This is called the bundle of $H$-structures of the principal $G$-bundle $P \rightarrow X$. The principal $H$-bundle structure determined by $P$ as manifold, $H$ a group acting by $\lambda$, and $H$ Str as base manifold shall be called the principal $H$-bundle $P^{H \text { Str }}$ induced by the principal $G$-bundle $P \rightarrow X$, on the manifold of $H$-structures $H$ Str.

Elements in a particular fiber $\pi_{H \operatorname{Str}}^{-1}(x)=H \operatorname{Str}_{x}$ are $H$-orbits $q=H p \in P / H$ contained in an specific $G$-orbit $P_{x} \subset P$. A section $q: x \in X \mapsto q_{x} \in H$ Str of the bundle $\pi_{H \text { Str }}: H \operatorname{Str} \rightarrow X$ can be seen as a smoothly varying choice of $H$-orbits $q_{x} \subset P_{x}$ for all possible $x \in X$, therefore as a choice of a smooth sub-bundle $Q \rightarrow X$ of the fiber bundle $P \rightarrow X$, where each fiber is a single $H$-orbit. Therefore, sections $q \in \Gamma(H \mathrm{Str})$ of the bundle of $H$ structures are simply sub-bundles of $P \rightarrow X$ whose fibers are orbits by the restricted action of the subgroup $H$. These are called reductions of the principal bundle $P$ to the subgroup $H$, or $H$-structures contained in $P$.

Consider $V^{\pi^{G}} P \subset T P$, the space of tangent vectors in $P$ that project as zero by $\mathrm{d} \pi^{G}: T P \rightarrow T X$. The action $\lambda_{g}$ on $P$ induces an action $\mathrm{d} \lambda_{g}$ on $V^{\pi^{G}} P$. The quotient manifold $\left(V^{\pi^{G}} P\right) / G$ is then a vector bundle on $P / G=X$, called adjoint bundle $\pi_{\mathrm{Ad}}$ : Ad $P \rightarrow X$. Giving an element $a_{x} \in \operatorname{Ad} P_{x}$ of this quotient over a point $x \in X$ is the same as giving a vertical vector at some point $p \in P_{x}$ together with all the vectors obtained by application of $\mathrm{d} \lambda_{g}$. This is the same as giving a vector field on the fiber $P_{x}$, that is invariant for all the automorphisms $\lambda_{g}: P_{x} \rightarrow P_{x}$ of this fiber. Any such a $G$-invariant vector field $a_{x}$ on a single fiber $P_{x}$ generates a 1-parameter flow, a family of automorphisms $\left(\exp \epsilon a_{x}\right): P_{x} \rightarrow P_{x}$ on the $G$-orbit $P_{x}$. All of them commute with all actions $\lambda_{g}$. Moreover, by definition of the flow, all tangent vectors:

$$
\left(\frac{\mathrm{d}}{\mathrm{d} \epsilon}\right)_{0}\left(\exp \epsilon a_{x}\right)\left(p_{x}\right) \in V_{p_{x}} P
$$

are a representative of the vector field $a_{x}$ on $P_{x}$. That is, all these tangent vectors project in $V P / G$ as the element $a_{x} \in V P / G=\operatorname{Ad} P$. 
In a similar way if we consider $V^{\pi^{H}} P$ the space of tangent vectors that project as zero by $\mathrm{d} \pi^{H}$, and the action $\lambda_{h}$ on $P$, we have an induced action by $\mathrm{d} \lambda_{h}$ on $V^{\pi^{H}} P$. The quotient manifold $\left(V^{\pi^{H}} P\right) / H$ is then a vector bundle on $P / H=H$ Str, which by definition represents the adjoint bundle Ad $P^{H \text { Str }} \rightarrow H$ Str of the principal $H$-bundle $\pi^{H}: P \rightarrow P / H$ (a bundle that we denote as $P^{H \text { Str }} \rightarrow H$ Str). Any element $a_{q} \in P_{q}^{H \text { Str }}$ on the fiber associated to $q \in H$ Str also induces a 1-parameter family of automorphisms exp $\epsilon a_{q}: Q_{x} \rightarrow Q_{x}$ on the $H$-orbit $Q_{x} \subset P_{x}$ determined by $q \in H \operatorname{Str}_{x}$. These automorphisms commute with each $\lambda_{h}$ for $h \in H$.

Proposition 2.1

Consider a principal $G$-bundle $P$ and for any closed subgroup $H \subseteq G$ the induced principal $H$-bundle $P^{H \text { Str }}$ on the manifold of $H$-structures. There exists an exact sequence of vector bundles on $H \mathrm{Str}$

$$
0 \hookrightarrow \operatorname{Ad} P^{H \operatorname{Str}} \rightarrow H \operatorname{Str} \times_{X} \text { Ad } P \rightarrow V H \operatorname{Str} \rightarrow 0
$$

Proof

Observe that $\pi^{H}: P \rightarrow P / H$ determines a natural projector $p r: H \operatorname{Str} \times_{X}\left(V^{\pi^{G}} P\right) / G \rightarrow V(P / H)$, that associates to each $H$-orbit $q_{x} \in H \operatorname{Str}_{x}$ and each $G$-covariant vertical vector field $A$ on the fiber $P_{x}$ the tangent vector at $q_{x} \in P / H$ obtained by $\pi^{H}$-projection $\mathrm{d} \pi^{H}\left(A_{p_{x}}\right) \in T_{q_{x}} H \mathrm{Str}$, for any representative $p_{x}$ of the $H$-orbit $q_{x}$. The condition that $A$ is $G$-covariant, and in particular $H$-covariant, shows that this mapping does not depend on the particular choice of $p_{x}$ in the $H$-orbit $q_{x}$. The fact that $\mathrm{d} \pi^{H}$ is linear and surjective shows that this morphism is also linear and surjective.

As $\pi^{G}=\pi^{H} \circ \pi_{H \text { Str }}$, there exists a natural inclusion $V^{\pi^{H}} P \subseteq V^{\pi^{G}} P$. There also exists a natural projection $V^{\pi^{H}} P \rightarrow P \rightarrow H \mathrm{Str}=P / H$. The combination of both leads to a morphism:

$$
f: V^{\pi^{H}} P \rightarrow H \operatorname{Str} \times_{X}\left(V^{\pi^{G}} P\right) / G=H \operatorname{Str} \times_{X} \operatorname{Ad} P
$$

We observe that two elements in $V^{\pi^{H}} P$ mapping to the same element in $H \operatorname{Str} \times_{X} \operatorname{Ad} P$ are necessarily one the image of the other by $\mathrm{d} \lambda_{g}$ for some $g \in G$ (because they determine the same element in $\left(V^{\pi^{G}} P\right) / G$ ), and that this $g \in G$ belongs in fact to the subgroup $H$ (because they determine the same element in $H$ Str $=P / H$ ). We conclude that fibers of $f$ are empty or a whole orbit in $V^{\pi^{H}} P$ by the action $\mathrm{d} \lambda_{h}$ for $h \in H$.

Therefore $f$ determines a natural immersion:

$$
i: \operatorname{Ad} P^{H \operatorname{Str}}=\left(V^{\pi^{H}} P\right) / H \hookrightarrow H \operatorname{Str} \times_{X} \operatorname{Ad} P
$$

We still have to prove that the exactness in the central term in the sequence:

$$
0 \hookrightarrow \operatorname{Ad} P^{H \operatorname{Str}} \rightarrow H \operatorname{Str} \times_{X} \operatorname{Ad} P \rightarrow V H \operatorname{Str} \rightarrow 0
$$

As elements in $V^{\pi^{H}} P$ project as zero into $H \mathrm{Str}=P / H$, in this sequence the image of $i$ is contained in the kernel of $p r$. Taking into account that we have vector bundles on $H$ Str with $\operatorname{ranks} \operatorname{dim} H, \operatorname{dim} G, \operatorname{dim} H \operatorname{Str}=$ $\operatorname{dim} G-\operatorname{dim} H$, and linear morphisms, we conclude that the image of $i$ must coincide with the kernel of $p r$ and this is an exact sequence.

\section{Corollary 2.1}

Consider the adjoint bundles $\operatorname{Ad} P \rightarrow X$ and $\operatorname{Ad} P^{H \text { Str }} \rightarrow H$ Str associated to the principal $G$-bundle $P \rightarrow X$ and $H$-bundle $P^{H \text { Str }} \rightarrow H$ Str. There exists a natural identification $V H \operatorname{Str} \simeq\left(H \operatorname{Str} \times_{X} \operatorname{Ad} P\right) / \operatorname{Ad} P^{H \text { Str }}$ and, for each $H$-structure $q: X \rightarrow H$ Str a natural identification $q^{*} V H \operatorname{Str} \simeq \operatorname{Ad} P / q^{*} \operatorname{Ad} P^{H \operatorname{Str}}$. The corresponding dual bundle can be identified as a sub-bundle $q^{*} V^{*} H \operatorname{Str} \subset \operatorname{Ad}^{*} P$ of linear forms on $\operatorname{Ad} P$ that vanish when applied to $q^{*} \operatorname{Ad} P^{H \operatorname{Str}} \subset \operatorname{Ad} P$.

\subsection{Euler-Poincaré equations for H-reduced fields on principal G-bundles}

Let $\pi: P \rightarrow X$ be a principal $G$-bundle and denote $\lambda_{g}$ the left-translation by any element $g \in G$. If we denote by $\pi_{J}: J P \rightarrow P$ the associated first jet bundle, the group $G$ acts on $J P$ by the induced mappings $j \lambda_{g}: J P \rightarrow J P$. 
An Ehresmann connection on the bundle $\pi: P \rightarrow X$ is any linear immersion $\chi: \pi^{*} T X \hookrightarrow T P$, section of the natural linear projector $\mathrm{d} \pi: T P \rightarrow \pi^{*} T X$. Giving a connection is then equivalent to giving a section $\chi: P \rightarrow J P$ of the associated first jet bundle (consult the theory of Ehresmann's connections in [23]).

The connection $\chi$ is called principal connection if it is invariant by the linear morphisms induced by $\lambda_{g}$ on the bundle $T P \otimes \pi^{*} T^{*} X$. Principal connections are equivalent to sections $\chi: P / G \rightarrow J P / G$ of the so-called bundle of principal connections $\pi_{\mathrm{CP}}: \mathrm{CP}=J P / G \rightarrow P / G=X$.

For any fixed Ehresmann connection on a bundle $\pi: P \rightarrow X$ there exists a natural (horizontal) lift of vector fields on $X$ to vector fields on $P$. To be precise, given a vector field $A: X \rightarrow T X$, it induces $\operatorname{Id} \times A: P \times{ }_{X} X \rightarrow$ $P \times_{X} T X=\pi^{*} T X$, which composed with $\chi$ determines $A^{\chi}: P=P \times_{X} X \rightarrow T P$, a vector field on $P$ called the horizontal lift of $A \in \Gamma(X, T X)$. For any pair of vector fields $A, B \in \Gamma(X, T X)$ the commutator $\left[A^{\chi}, B^{\chi}\right] \in$ $\Gamma(P, T P)$ of the corresponding horizontal lifts $A^{\chi}, B^{\chi}$ can be compared to the horizontal lift $[A, B]^{\chi}$ of the commutator $[A, B] \in \Gamma(X, T X)$. Both of these fields on $P$ project to the manifold $X$ as $[A, B] \in \Gamma(X, T X)$ and the difference is then a vertical vector field:

$$
\text { Curv } \chi:(A, B) \in \Gamma(X, T X) \times \Gamma(X, T X) \mapsto\left[A^{\chi}, B^{\chi}\right]-[A, B]^{\chi} \in \Gamma(P, V P)
$$

In fact, one can prove that $\operatorname{Curv} \chi$ is a bilinear alternating form, leading then to $\operatorname{Curv} \chi \in \Gamma\left(\pi^{*}\left(\Lambda^{2} T^{*} X\right) \otimes V P\right)$, called the curvature tensor associated to the Ehresmann connection $\chi$.

In the case that the connection on $P$ is a principal connection (invariant by translations $\lambda_{g}$ ), its associated curvature tensor will also be invariant by translations and can be described as

$$
\operatorname{Curv} \chi \in \Gamma\left(\Lambda^{2} T^{*} X \otimes \operatorname{Ad} P\right)
$$

a 2-form on the base manifold, with values on the adjoint bundle $\pi_{\mathrm{Ad}}$ : $\mathrm{Ad} P=V P / G \rightarrow X$. A connection is called flat when its curvature vanishes.

As a remark, observe that $\pi^{H} \circ \pi_{J}: J P \rightarrow P / H$ and $\pi^{G}: J P \rightarrow(J P) / G$ determine a smooth surjective mapping $J P \rightarrow(P / H) \times_{X}(J P / G)$. Moreover, two elements in the same fiber by this mapping must differ by some transformation $j \lambda_{g}$ (because they determine the same element in $(J P) / G$ ) and necessarily for some $g \in H$ (because they determine the same element in $P / H$ ). Fibers of the morphism are precisely orbits on $J P$ by the action $j \lambda_{h}$ for $h \in H$. There exists a naturally induced isomorphism defined on the quotient manifold $J P / H$ :

$$
(J P) / H \simeq(P / H) \times_{X}(J P) / G=H \operatorname{Str} \times_{X} \mathrm{CP}
$$

\section{Definition 2.4}

For any principal $G$-bundle $\pi: P \rightarrow X$ and closed subgroup $H \subseteq G$, we call local $H$-reduced field any local section of the bundle $Y=J P / H \simeq H \operatorname{Str} \times{ }_{X} \mathrm{CP}$. We call $H$-reduced Lagrangian function any function $\ell$ on some open sub-bundle of $J P / H=Y \rightarrow X$, or equivalently, an $H$-invariant function on some open sub-bundle of $J P \rightarrow X$.

We recall now how a principal connection determines parallelism notions on associated bundles. Consider any manifold $E \rightarrow X$ and smooth action $a c:(e, g) \in E \times G \mapsto e g \in E$ of the Lie group $G$ on the right on $E$. Using the left action $g \cdot(e, p)=\left(e g^{-1}, g p\right)$ on the manifold $E \times P$, there exists a quotient manifold $Q^{E}=(E \times P) / G$, called the associated bundle for the principal bundle $P$ and the $G$-space $E$. For a given principal connection $\chi$ on $P$, and any associated bundle, there exists an induced Ehresmann connection $\chi^{Q}$ on the bundle $\pi_{Q}: Q^{E} \rightarrow X$.

We recall that a connection $\chi^{Q}$ on a bundle $\pi_{Q}: Q \rightarrow X$ is a linear immersion $\chi^{Q}: \pi_{Q}^{*} T X=Q \times_{X} T X \hookrightarrow T Q$, section of the natural linear projector $\mathrm{d} \pi_{Q}: T Q \rightarrow Q \times_{X} T X$. For any section $q \in \Gamma(Q)$ we may compute the difference of $\chi_{q}^{Q}=\chi^{Q} \circ q \in \Gamma\left(q^{*} T Q \otimes T^{*} X\right)$ with $\mathrm{d} q \in \Gamma\left(q^{*} T Q \otimes T^{*} X\right)$. As both linear morphisms are sections of $\mathrm{d} \pi_{Q}: T X \rightarrow q^{*} T Q$, the difference is a linear mapping $T X \rightarrow q^{*} V Q$ and determines a notion of covariant derivative of $q \in \Gamma(Q)$ with respect to the connection $\chi^{Q}$ :

$$
\mathrm{d}_{x}^{\chi^{Q}} q=\mathrm{d}_{x} q-\chi_{q(x)}^{Q} \in V_{q(x)} Q \otimes T_{x}^{*} X
$$

We say $q \in \Gamma(Q)$ is $\chi$-parallel if $\mathrm{d}^{\chi} q=0$. 
Using $E=G / H$ (with left cosets $G / H=\{H g\}_{g \in G}$ ) and the natural action on the right of the group $G$ on this space, from $\chi \in \Gamma(\mathrm{CP})$ we obtain a connection on the bundle of $H$-structures $\pi_{H \mathrm{Str}}: H \mathrm{Str}=E \times P=P / H \rightarrow$ $X$. A principal connection $\chi$ determines a connection $\chi^{H \text { Str }}$ on this bundle and we have a notion of covariant derivative of any local $H$-structure $q \in \Gamma(H \mathrm{Str})$, with respect to the principal connection $\chi$.

$$
\mathrm{d}_{x}^{\chi} q=\mathrm{d}_{x} q-\chi_{q(x)}^{H \operatorname{Str}} \in V_{q(x)} H \operatorname{Str} \otimes T_{x}^{*} X
$$

\section{Remark 2.1}

One should observe that any local field $p \in \Gamma(P)$ naturally induces, by projection $\pi^{H}: J P \rightarrow J P / H$, a local $H$ reduced field. However, there may exist local $H$-reduced fields $(q, \chi) \in \Gamma\left(H \operatorname{Str} \times_{X} \mathrm{CP}\right)$ that are not projection of any local field on $P$. There are differential and topological obstructions in this regard [10]. Whenever the $H$ reduced field $(q, \chi)$ is obtained projecting a jet extension $j p \in \Gamma(J P)$, the principal connection component $\chi$ is flat (has vanishing curvature), and the $H$-structure component $q \in \Gamma(H \mathrm{Str})$ is $\chi$-parallel, that is, $\mathrm{d}^{\chi} q=0$. In fact, this condition can be stated saying that the principal connection $\chi$ on the principal $G$-bundle $P$ is reducible to the principal sub- $H$-bundle $Q \rightarrow X$ determined by $q \in \Gamma(H \mathrm{Str})$. That is, horizontal lifts at points $p_{x} \in Q_{x} \subset P_{x}$ give tangent vectors belonging to $T_{q_{x}} Q_{x} \subset T_{q_{x}} P_{x}$.

In particular, using $E=\operatorname{Lie} G$ with the right adjoint action, the associated bundle is the adjoint bundle and any principal connection $\chi$ on $P$ induces an Ehresmann connection $\chi^{\mathrm{Ad}}$ on the adjoint bundle $\pi_{\mathrm{Ad}}: \operatorname{Ad} P \rightarrow X$. We get a notion of covariant derivative of any local section of the adjoint bundle $a \in \Gamma(\operatorname{Ad} P)$ with respect to the principal connection $\chi$ :

$$
\mathrm{d}_{x}^{\chi} a=\mathrm{d}_{x} a-\chi_{a(x)}^{\mathrm{Ad}} \in V_{a(x)} \operatorname{Ad} P \otimes T_{x}^{*} X \simeq \operatorname{Ad} P_{x} \otimes T_{x}^{*} X
$$

where the identification $V_{a(x)} \operatorname{Ad} P \simeq \operatorname{Ad} P_{x}$ is using the natural identification of tangent vectors at any point $a(x)$ of a linear space $\operatorname{Ad} P_{x}$ with elements of the linear space itself.

\section{Definition 2.5}

A local $H$-reduced field $(q, \chi) \in \Gamma\left(H \operatorname{Str} \times{ }_{X} \mathrm{CP}\right)$ is called admissible if $\chi$ has vanishing curvature and $q$ is $\chi$ parallel (equivalently, the connection $\chi$ is flat and reducible to the specific $H$-structure $q$ ). This determines the following constraint submanifold on $J\left(H \operatorname{Str} \times{ }_{X} \mathrm{CP}\right)$ :

$$
\mathcal{S}=\left\{\left(j_{x}^{1} q, j_{x}^{1} \chi\right):(\operatorname{Curv} \chi)_{x}=0, \mathrm{~d}_{x}^{\chi} q=0\right\} \subset J\left(H \operatorname{Str} \times{ }_{X} \mathrm{CP}\right)
$$

This submanifold $\mathcal{S}$ determines a space of admissible jet configurations, the first element needed in definition 2.2 to characterize a constrained variational principle.

Finally for any admissible local $H$-reduced field $(q, \chi) \in \Gamma(Y)$ we may consider two linear operators:

$$
\begin{gathered}
\mathcal{P}_{q}: a \in \Gamma(\operatorname{Ad} P) \mapsto \mathcal{P}_{q}(a)=[a] \in \Gamma\left(\operatorname{Ad} P /\left(q^{*} \operatorname{Ad} P^{H \operatorname{Str}}\right)\right) \simeq \Gamma\left(q^{*} V H \operatorname{Str}\right) \\
\mathcal{P}_{\chi}: a \in \Gamma(\operatorname{Ad} P) \mapsto \mathcal{P}_{\chi}(a)=\mathrm{d}^{\chi} a \in \Gamma\left(\operatorname{Ad} P \otimes T^{*} X\right) \simeq \Gamma\left(\chi^{*} V \mathrm{CP}\right)
\end{gathered}
$$

here we are using that $\mathrm{CP}$ is an affine bundle modelled on $\operatorname{Ad} P \otimes T^{*} X$, and we are considering the principal $H$-bundle $\pi^{H}: P \rightarrow P / H=H$ Str, that we denote as $P^{H \text { Str }}$, its associated adjoint bundle $\operatorname{Ad} P^{H \text { Str }} \rightarrow H$ Str and the projection $a \mapsto[a]$ in the natural exact sequence:

$$
0 \rightarrow q^{*} \operatorname{Ad} P^{H \operatorname{Str}} \rightarrow \operatorname{Ad} P \rightarrow q^{*} V H \operatorname{Str} \rightarrow 0
$$

Using these operators $\mathcal{P}_{q}, \mathcal{P}_{\chi}$ (the first one a linear projection and the second one a first order differential operator) we have corresponding spaces of admissible variations:

\section{Definition 2.6}

For any admissible local $H$-reduced field $(q, \chi) \in \Gamma\left(H \operatorname{Str} \times_{X} \mathrm{CP}\right)$ we call space of admissible infinitesimal variations at $(q, \chi)$ the linear space:

$$
\mathcal{A} \mathcal{V}_{(q, \chi)}=\left\{\left(\mathcal{P}_{q}(a), \mathcal{P}_{\chi}(a)\right): a \in \Gamma^{c}(\operatorname{Ad} P)\right\} \subset \Gamma^{c}\left(q^{*} V H \operatorname{Str}\right) \oplus \Gamma^{c}\left(\chi^{*} V \mathrm{CP}\right)=\Gamma^{c}\left((q, \chi)^{*} V\left(H \operatorname{Str} \times{ }_{X} \mathrm{CP}\right)\right)
$$


As indicated in [10] any element $\left(\delta_{q}, \delta_{\chi}\right) \in \mathcal{A} \mathcal{V}_{(q, \chi)}$ has an associated infinitesimal contact transformation $j^{1}\left(\delta_{q}, \delta_{\chi}\right) \in \Gamma\left(J\left(H \operatorname{Str} \times{ }_{X} \mathrm{CP}\right)\right)$ that is tangential to the constraint submanifold $\mathcal{S} \subset J\left(H \mathrm{Str} \times{ }_{X} \mathrm{CP}\right)$. The constraint submanifold $\mathcal{S}$ and the family of spaces of admissible infinitesimal variations $\mathcal{A} \mathcal{V}_{(q, \chi)}$ serve as compatible data to define a constrained variational problem on $H \operatorname{Str} \times{ }_{X} \mathrm{CP}$, as described in definition 2.2.

The constrained variational problem on $H$-reduced fields is finally fixed when we consider some $H$-reduced Lagrangian density, a function on $\mathcal{S}$ that we will consider as determined by the values of the field and not of its jet extension $j_{x}^{1}(q, \chi) \in \mathcal{S} \subset J\left(H \operatorname{Str} \times{ }_{X} \mathrm{CP}\right)$. We shall restrict our variational problems to the following type of Lagrangian functions:

\section{Definition 2.7}

We call zero-order $H$-reduced Lagrangian function any function $\ell: H \operatorname{Str} \times{ }_{X} \mathrm{CP} \rightarrow \mathbb{R}$. Giving a zero-order $H$ reduced Lagrangian function is the same as giving a first order Lagrangian function $\mathcal{L}: J P \rightarrow \mathbb{R}$ that is invariant by $j^{1} \lambda_{h}$, for each $h \in H$ (Recall that $J P / H \simeq H \operatorname{Str} \times{ }_{X} \mathrm{CP}$ ).

Zero-order $H$-reduced Lagrangian functions determine a Lagrangian function $\mathcal{S} \rightarrow \mathbb{R}$, as demanded in definition 2.2 of constrained variational problems. In this situation we have the following fundamental result:

Theorem 2.2 ([10])

Let $\pi: P \rightarrow X$ be a principal $G$-bundle on a manifold $X$ with volume element $\operatorname{vol}_{X}$. Let $H \subseteq G$ be a closed subgroup, and denote by $\pi^{H}: J P \rightarrow H \operatorname{Str} \times{ }_{X} \mathrm{CP}$ the natural quotient morphism. Let $\ell: H \operatorname{Str} \times{ }_{X} \mathrm{CP} \rightarrow \mathbb{R}$ be a zero-order $H$-reduced Lagrangian function and $\mathcal{L}=\ell \circ \pi^{H}: J P \rightarrow \mathbb{R}$ the associated first order Lagrangian function.

For any local field $p \in \Gamma(P)$, and for the induced local $H$-reduced field $\pi^{H} \circ j^{1} p=(q, \chi) \in \Gamma\left(H \operatorname{Str} \times{ }_{X} \mathrm{CP}\right)$, the following are equivalent:

1. The local field $p$ is critical for the variational problem with fixed boundary variations and Lagrangian function $\mathcal{L}$.

2. The local field $p \in \Gamma(P)$ satisfies the system of Euler-Lagrange equations $0=\mathcal{E} \mathcal{L}(p)$ for $\mathcal{L}: J P \rightarrow \mathbb{R}$, where $\mathcal{E} \mathcal{L}(p)$ is described by (4).

3. The local $H$-reduced field is critical for the constrained variational problem described on $H \operatorname{Str} \times{ }_{X} \mathrm{CP}$ by the constraint submanifold $\mathcal{S}$, admissible infinitesimal variations $\mathcal{A} \mathcal{V}_{(q, \chi)}$, and Lagrangian function $\ell \circ \pi_{J}: J\left(H \operatorname{Str} \times{ }_{X} \mathrm{CP}\right) \rightarrow \mathbb{R}$.

4. The $H$-reduced field $(q, \chi) \in \Gamma\left(H \operatorname{Str} \times{ }_{X} \mathrm{CP}\right)$ satisfies the system of Euler-Poincaré equations $0=\mathcal{E} \mathcal{P}(q, \chi)$ for $\ell: H \operatorname{Str} \times{ }_{X} \mathrm{CP} \rightarrow \mathbb{R}$, where $\mathcal{E} \mathcal{P}(q, \chi)$ is described by:

$$
\mathcal{E P}(q, \chi)=\operatorname{div}_{\chi}\left(\frac{\partial \ell}{\partial \chi}(q, \chi)\right)-\mathcal{P}_{q}^{*}\left(\frac{\partial \ell}{\partial q}(q, \chi)\right) \in \Gamma\left(\operatorname{Ad}^{*} P\right)
$$

In this result $\partial \ell / \partial \chi \in \Gamma\left(\chi^{*} V^{*} \mathrm{CP}\right) \simeq \Gamma\left(\mathrm{Ad}^{*} P \otimes T X\right)$ (recall that $\mathrm{CP}$ is an affine bundle on $\left.\operatorname{Ad} P \otimes T^{*} X\right)$, $\partial \ell / \partial q \in \Gamma\left(\left(\operatorname{Ad} P / q^{*} \operatorname{Ad} P^{H \operatorname{Str}}\right)^{*}\right)$ (recall that $\left.q^{*} V H \operatorname{Str} \simeq \operatorname{Ad} P / q^{*} \operatorname{Ad} P^{H \operatorname{Str}}\right), \mathcal{P}_{q}^{*}$ is the natural immersion $\Gamma\left(\left(\operatorname{Ad} P / q^{*} \operatorname{Ad} P^{H \operatorname{Str}}\right)^{*}\right) \subset \Gamma\left(\operatorname{Ad}^{*} P\right)$ as the subspace of linear forms that vanish on $q^{*} \operatorname{Ad} P^{H \operatorname{Str}} \subset \operatorname{Ad} P$, and finally the divergence operator $\operatorname{div}_{\chi}: \Gamma\left(\operatorname{Ad}^{*} P \otimes T X\right) \rightarrow \Gamma\left(\operatorname{Ad}^{*} P\right)$ associated to $\chi$ represents the differential operator adjoint to $\mathrm{d}^{\chi}$, in the sense:

$$
\operatorname{div}_{\chi}(\theta) \otimes \operatorname{vol}_{X}=\mathrm{d}^{\chi}\left(i_{\theta} \operatorname{vol}_{X}\right)
$$

here $i_{\theta} \operatorname{vol}_{X}$ stands for the Ad $P$-valued $n$-1-alternating form obtained by contraction of $\theta$ with the volume element $X$ and $\mathrm{d}^{\chi}$ stands for the covariant differential on alternating forms with values on $\operatorname{Ad} P$ and on $\operatorname{Ad}^{*} P$ obtained as extension of $\mathrm{d}^{\chi}: \Gamma(\operatorname{Ad} P) \rightarrow \Gamma\left(\operatorname{Ad} P \otimes T^{*} X\right)$.

This theorem relates the variational problem with fixed boundary variations determined by the Lagrangian function $\mathcal{L}=\ell \circ \pi^{H}$ on $J P$, with the constrained variational problem on $H \operatorname{Str} \times_{X} \mathrm{CP}$ with admissible infinitesimal variations $\mathcal{A} \mathcal{V}_{(q, \chi)}$, determined by the Lagrangian function $\ell \circ \pi_{J}: \mathcal{S} \rightarrow \mathbb{R}$ on the constraint submanifold in $J\left(H \mathrm{Str} \times{ }_{X} \mathrm{CP}\right)$. We stress, however, that this does not imply that both variational problems are equivalent. There may exist admissible reduced fields that are not obtained as projection of a jet extension for any section of the principal bundle. 


\subsection{Discretization through Forward difference operators}

We aim to obtain an appropriate discretization of the variational problems with constraints on local $H$ reduced fields. That is, if we have a principal $G$-bundle $\pi: P \rightarrow X$ and an $H$-reduced Lagrangian function $\ell: H \operatorname{Str} \times{ }_{X} \mathrm{CP} \rightarrow \mathbb{R}$, we want to substitute the infinite-dimensional space of smooth $H$-reduced fields by some finite-dimensional (at least locally) space of discrete $H$-reduced fields. We also need to relate $H$-reduced fields with a discrete counterpart, and to derive a variational principle on these discrete fields from the given smooth $H$-reduced Lagrangian function $\ell$.

Discretization mechanisms substitute partial derivatives of a smooth field with some kind of difference between the configurations of the field at two given points. In this process it is desirable that the main symmetries and geometrical properties of the smooth theory are retained in the discretized formulation. This was achieved in [6], where the corresponding discrete notions and discretization mechanism were introduced using forward difference operators in a covariant way. In the reduction and discretization of elements of the variational theory on a principal $G$-bundle $P$, a relevant object was Ehresmann's groupoid of fiber-to-fiber endomorphisms.

On a principal bundle $\pi: P \rightarrow X$ the natural mapping $(p, g) \in P \times G \mapsto(p, g p) \in P \times{ }_{X} P$ establishes a diffeomorphism, whose inverse mapping takes the form $(p, \bar{p}) \in P \times{ }_{X} P \mapsto\left(p, \bar{p} p^{-1}\right) \in P \times G$ determining the group difference map $(p, \bar{p}) \in P \times{ }_{X} P \mapsto \bar{p} p^{-1} \in G$, characterized by $\left(\bar{p} p^{-1}\right) p=\bar{p}$. This way of computing differences, however, can be performed only on pairs belonging to the same $G$-orbit and the difference is not preserved when we apply left translations $\lambda_{g}: P \rightarrow P$ on the pair.

For any pair of elements $p_{0} \in P_{x_{0}}, p_{1} \in P_{x_{1}}$, if we want to fix some $G$-covariant morphism $\psi: P \rightarrow P$ such that $\psi\left(p_{0}\right)=p_{1}$, there might be several choices, but on the $x_{0}$-fiber the choice is totally determined, indeed for any $\bar{p} \in P_{x_{0}}$ we may write $\bar{p}=\left(\bar{p} p_{0}^{-1}\right) p_{0}$ and consequently $\psi(\bar{p})=\left(\bar{p} p_{0}^{-1}\right) \psi\left(p_{0}\right)=\left(\bar{p} p_{0}^{-1}\right) p_{1}$ must be the image of any $\bar{p} \in P_{x_{0}}$.

\section{Definition 2.8}

For any pair of elements $p_{0} \in P_{x_{0}}, p_{1} \in P_{x_{1}}$ we call fiber-to-fiber endomorphism induced by $\left(p_{0}, p_{1}\right)$, denoting it as $p_{0}^{-1} p_{1}$, the unique $G$-covariant mapping $\psi: P_{x_{0}} \rightarrow P$ whose domain is the $G$-orbit of $p_{0}$ and such that $\psi\left(p_{0}\right)=p_{1}$. This mapping takes the form $\bar{p} \in P_{x_{0}} \mapsto\left(\bar{p} p_{0}^{-1}\right) p_{1} \in P_{x_{1}}$, for the element $\bar{p} p_{0}^{-1} \in G$ defined as the group difference of $p_{0}, \bar{p} \in P_{x_{0}}$, and has the following properties:

$$
p_{x} q_{x}^{-1}=e \Leftrightarrow p_{x}=q_{x}, \quad\left(q_{x} p_{x}^{-1}\right) p_{x}=q_{x}, \quad\left(g q_{x}\right) p_{x}^{-1}=g\left(q_{x} p_{x}^{-1}\right), \quad p_{x} q_{x}^{-1}=\left(q_{x} p_{x}^{-1}\right)^{-1}
$$

\section{Definition 2.9}

We call fiber-to-fiber endomorphism on the principal $G$-bundle $\pi: P \rightarrow X$ any $G$-covariant morphism $\psi: P_{x} \rightarrow P$ (we mean $\psi \circ \lambda_{g}=\lambda_{g} \circ \psi$ for each $g \in G$ ) whose domain is a single fiber $P_{x} \subseteq P$.

The mapping $s:$ End $P \rightarrow X$ taking a fiber-to-fiber endomorphism into its domain $\operatorname{Dom}(\psi)=P_{x} \in P / G \simeq X$ is called the source mapping on End $P$. By $G$-covariance, the image of the $G$-orbit $P_{x}$ must be another $G$-orbit. The mapping $t$ : End $P \rightarrow X$ taking a fiber-to-fiber endomorphism into its image $\operatorname{Img}(\psi)=P_{x_{1}} \in P / G \simeq X$ is called the target mapping on End $P$.

For any fiber-to-fiber endomorphism $\psi$ and for any element in its source fiber $p_{0} \in P_{s(\psi)}$, the image of $p_{0}$ by $\psi$ shall be denoted as $p_{0} \psi$. This element lies in $P_{t(\psi)} \subset P$.

\section{Remark 2.2}

As proved in [6], the mapping $\left(p_{0}, p_{1}\right) \in P \times P \mapsto p_{0}^{-1} p_{1} \in$ End $P$ that transforms any pair into its associated fiber-to-fiber endomorphism is surjective and its fibers are the orbits in $P \times P$ by the diagonal action $\lambda_{g} \times \lambda_{g}$. Therefore there exists an identification $(P \times P) / G \simeq$ End $P$ and the set End $P$ has a natural smooth structure. The projectors $s$ : End $P \rightarrow X$ and $t$ : End $P \rightarrow X$ are smooth and $(s, t):$ End $P \rightarrow X \times X$ is called Ehresmann's gauge groupoid.

There is a natural product (composition) of any fiber-to-fiber endomorphism with target $x_{1}$ with any fiber-tofiber endomorphism with source $x_{1}$, determining a smooth groupoid product $\left(\psi_{0}, \psi_{1}\right) \in$ End $P \times_{(t, s)}$ End $P \mapsto$ $\psi_{1} \circ \psi_{0} \in$ End $P$. The manifold End $P$ has a groupoid structure using $(s, t)$ : End $P \rightarrow X \times X$ as anchor mapping and $\circ$ as product. 
For notational convention the image of $p \in P_{x}$ by $\psi \in(\text { End } P)_{x}$ will be denoted $p \psi \in P_{t(\psi)}$ and the composition $\psi_{1} \circ \psi_{0}$ as $\psi_{0} \cdot \psi_{1}$. In this notation - represents the reverse product structure on the set End $P$. We have, moreover:

$$
\begin{aligned}
& p_{0}\left(p_{0}^{-1} p_{1}\right)=p_{1}, \quad \pi\left(p_{0} \psi_{01}\right)=t\left(\psi_{01}\right), \quad p_{0} \psi_{01}=p_{0} \Leftrightarrow \psi_{01}=\operatorname{Id}_{\pi\left(p_{0}\right)} \\
& q_{0}\left(p_{0}^{-1} p_{1}\right)=\left(q_{0} p_{0}^{-1}\right) p_{1}, \quad(s, t)\left(p_{0}^{-1} p_{1}\right)=\left(\pi\left(p_{0}\right), \pi\left(p_{1}\right)\right), \quad\left(p_{0}^{-1} p_{1}\right)^{-1}=p_{1}^{-1} p_{0} \\
& \left(g p_{0}\right)^{-1}\left(g p_{1}\right)=p_{0}^{-1} p_{1} \text {, } \\
& \left(g p_{0}\right) \psi_{01}=g\left(p_{0} \psi_{01}\right), \quad p_{0}^{-1}\left(p_{1} \psi_{12}\right)=\left(p_{0}^{-1} p_{1}\right) \psi_{12} \\
& \left(p_{0} \psi_{01}\right) \psi_{12}=p_{0}\left(\psi_{01} \psi_{12}\right) \text {, } \\
& \left(q_{0} \psi_{01}\right)\left(p_{0} \psi_{01}\right)^{-1}=q_{0} p_{0}^{-1}, \quad\left(\psi_{01} \psi_{12}\right)^{-1}=\psi_{12}^{-1} \psi_{01}^{-1} \\
& (s, t)\left(\psi_{01} \psi_{12}\right)=\left(s\left(\psi_{01}\right), t\left(\psi_{12}\right)\right), \quad p_{0}^{-1} p_{1}=\operatorname{Id}_{P_{\pi\left(p_{0}\right)}} \Leftrightarrow p_{0}=p_{1}, \quad p_{0} \psi_{01}=p_{0} \Leftrightarrow \psi=\operatorname{Id}_{s(\psi)}
\end{aligned}
$$

The isotropy group bundle associated to a groupoid bundle $(s, t)$ : End $P \rightarrow X \times X$ is defined as the subset of elements that have a coincident source and target. This determines a bundle Gau $P \rightarrow X$ called the gauge group bundle, whose elements are $G$-covariant automorphisms defined on a single fiber $P_{x}$. Recall that the flow associated to $G$-invariant vector fields on a fiber is always given by $G$-covariant automorphisms of this fiber. Therefore the flow $\epsilon \in \mathbb{R} \mapsto \phi_{\epsilon} \in \operatorname{Aut}\left(P_{x}\right)$ of $a_{x} \in \operatorname{Ad} P_{x}$ takes values in the gauge group bundle, and determines an exponential mapping $\left(\epsilon, a_{x}\right) \in \mathbb{R} \times \operatorname{Ad} P_{x} \mapsto \exp \epsilon a_{x} \in \operatorname{Gau} P_{x}$ that allows to interpret $\operatorname{Ad} P_{x}$ as the Lie algebra of the Lie group Gau $P_{x}$.

Discretization will replace partial derivatives at a single point, with a sample of configurations at several points. This leads to a pair of relevant definitions:

Definition 2.10

For any set $X$ and each $k \in \mathbb{N}$ we denote $X^{\times k}=X \times \ldots \times X$ the direct product of $k+1$ copies of the set $X$. Hence $X^{\times 0}=X, X^{\times 1}=X \times X$ and so on. Elements in $X^{\times k}$ can be seen as finite ordered sequences in $X$ consisting of $k+1$ terms. An ordered sequence $\left(x_{0}, x_{1}, \ldots, x_{k}\right) \in X^{\times k}$ without repeated terms shall be called an ordered abstract simplex (being an abstract simplex any finite subset of $X$ with exactly $k+1$ elements).

In a similar way, for any bundle $s: E \rightarrow X$ we denote by $E^{\times_{s} k}=E \times{ }_{s} \ldots \times{ }_{s} E$ the fibered product of $k+1$ copies of the bundle $s: E \rightarrow X$. Elements in $E^{{ }^{\times}}{ }^{k}$ can be seen as a point $x \in X$ together with a finite ordered sequence $\left(e_{0}, e_{1}, \ldots, e_{k}\right)$ consisting of $k+1$ terms on the fiber $E_{x}$ associated to the given point, that is, for the projection $s: E^{\times{ }_{s} k} \rightarrow X$ the fiber $\left(E^{\times{ }_{s} k}\right)_{x}$ is simply $\left(E_{x}\right)^{\times k}$.

One of the most relevant results in [6] is that fixing a locally defined mapping $\Delta_{G}$ : End $P \rightarrow T P / G$ with specific particular properties (projectable faithful reduced forward difference operator), delivers all the mechanisms that we shall use to describe a discrete $H$-reduced variational problem. Specifically from $\Delta_{G}$ one may derive:

- An open domain $\widetilde{X} \subset X^{\times n}$ (domain of regular facet configurations) and a smooth function $\widetilde{\text { vol }}: \widetilde{X} \rightarrow \mathbb{R}$ (the discrete volume function) associated to any volume form $\operatorname{vol}_{X} \in \Omega^{n}(X)$.

- An open domain $\widetilde{J P} \subset P^{\times n}$ (discrete jet space) projecting to $\widetilde{X}$ by $\pi^{\times n}$ and an injective local diffeomorphism $J_{X}^{P}: \widetilde{J P} \rightarrow J P \times_{\left(j \pi, \pi_{0}\right)} X^{\times n}$ (forward Jacobi operator) relating this domain with a corresponding open domain on the pull-back of the jet bundle $j \pi: J P \rightarrow X$ by the projector $\pi_{0}:\left(x_{0}, \ldots, x_{n}\right) \in X^{\times n} \mapsto x_{0} \in X$. This determines a smooth function $\mathcal{L}_{d}: \widetilde{J P} \rightarrow \mathbb{R}$ (the discrete Lagrangian function) associated to any Lagrangian density $\mathcal{L}_{\operatorname{vol}}$, for $\mathcal{L} \in \mathcal{C}^{\infty}(J P)$.

- An open domain $\widetilde{\mathrm{CP}} \subset(\text { End } P)^{\times_{s} n-1}$ (discrete connection space) projecting to $\widetilde{X}$ by $\left(s, t^{\times n-1}\right)$ and an injective local diffeomorphism $J_{\mathrm{CP}}: \widetilde{\mathrm{CP}} \rightarrow \mathrm{CP} \times_{\left(\pi_{\mathrm{CP}}, \pi_{0}\right)} X^{\times n}$ (reduced forward jacobi operator) relating this domain with a corresponding open domain on the pull-back of the bundle of principal connections $\pi_{\mathrm{CP}}: \mathrm{CP} \rightarrow X$ by the projector $\pi_{0}: X^{\times n} \rightarrow X$. This determines a smooth function $\ell_{d}: H \operatorname{Str} \times{ }_{X} \widetilde{\mathrm{CP}} \rightarrow \mathbb{R}$ (the discrete $H$-reduced Lagrangian function) associated to any $H$-reduced Lagrangian density $\ell \operatorname{vol}_{X}$ for $\ell \in \mathcal{C}^{\infty}\left(H \operatorname{Str} \times{ }_{X} \mathrm{CP}\right)$.

Moreover, both the forward jacobi and reduced forward jacobi operators are covariant with respect to the naturally induced actions of any gauge transformation $\phi: P \rightarrow P$ on the bundles $P, J P, H$ Str, CP and End $P$ (see [6]). 


\section{Discretization of space}

The foundations of differential calculus on abstract manifolds lie on choices of local charts, that is, locally defined topological immersions $x: \mathbb{R}^{n} \hookrightarrow X$, which may be restricted to open hypercubic domains $R \subseteq \mathbb{R}^{n}$. Stating that a point $x \in X$ has coordinates $r=\left(r_{1}, \ldots, r_{n}\right) \in R \subset \mathbb{R}^{n}$ in this local chart is the same as stating that $x=x\left(r_{1}, \ldots, r_{n}\right)$. Any function $f: X \rightarrow \mathbb{R}$ can be expressed in a local chart as a real function in $n$ real variables $\bar{f}\left(r_{1}, \ldots, r_{n}\right)=f \circ x: \mathbb{R}^{n} \rightarrow \mathbb{R}$, defined on an open subset and differential calculus is then performed on these real functions with several real variables. A geometrical treatment of differential calculus warrants that even though computations depend on local coordinates, we may define notions with an intrinsic meaning, independent of a possible change of one local coordinate chart to another using as coordinate transformations any diffeomorphism between any two arbitrary open subsets on $\mathbb{R}^{n}$.

To obtain a difference calculus on an abstract $n$-dimensional manifold a classical choice is to substitute the smooth local chart and fix a cartesian grid on the manifold, a particular (possibly locally defined) immersion $x: \mathbb{Z}^{n} \hookrightarrow X$ that associates to each vertex $v \in \mathbb{Z}^{n}$ a node $x(v) \in X$. In this way, for any function $f \in \mathcal{C}^{\infty}(X)$ we have a discrete counterpart $f_{d}=f \circ x$ and the corresponding difference notions at any node $\left(\Delta_{i} f_{d}\right)(v)=$ $f_{d}\left(t_{i}(v)\right)-f_{d}(v)$ where for each $i=1 \ldots n, t_{i}: \mathbb{Z}^{n} \rightarrow \mathbb{Z}^{n}$ stands for the unit translation in the $i$-th direction of $\mathbb{Z}^{n}$.

For the purposes of variational calculus, where we will deal with Lagrangian densities and connections, we must extend our interest to include also edges and facets and not just vertices. That is, we will not work with a simple set of vertices but with a richer structure, that of an abstract cellular complex [4, 5]. More specifically, in order to take advantage of (reduced) forward jacobi operators we shall work on a simple structure: abstract simplicial complexes $\mathcal{V}$ modelled on $\mathbb{Z}^{n}$.

\section{Definition 3.1}

Consider the integer interval $[N]=\{1, \ldots, N\} \subset \mathbb{Z}$. We call $V=[N] \times[N] \times \ldots \times[N] \subset \mathbb{Z}^{n}$ the $n$-dimensional array with diameter $N$. We call array with diameter $N$ on an $n$-dimensional manifold $X$ any immersion $x: V \hookrightarrow X$.

Elements in the $n$-dimensional array of diameter $N$ are points $\left(k_{1}, \ldots, k_{n}\right)$, where $k_{i} \in\{1, \ldots, N\}, \forall i=1 \ldots n$. An array with diameter $N$ on $X$ is therefore a choice of $N^{n}$ points on $X$, indexed as $\left(x_{v}\right)_{v \in V}$ for array values $v=\left(k_{1}, \ldots, k_{n}\right) \in V$. To make a distinction, we shall talk of vertices when using elements of the $n$-dimensional array $V$ and nodes when we use points $x_{v} \in X$, in the image of some array on $X$.

\section{Remark 3.1}

For simplicity we shall assume that a given total order is fixed on the set $V$, for example the lexicographical order. In this way, subsets of $V$ can be identified with monotone sequences $\left(v_{0}, v_{1}, \ldots, v_{m}\right)$ on $V$, and subsets of a set can be given as subsequences $\left(v_{j_{0}}, v_{j_{1}}, \ldots, v_{j_{k}}\right)$, where $0 \leq j_{0}<j_{1}<\ldots<j_{k} \leq m$.

To introduce a variational formalism for sections of a discrete bundle $Y_{d} \rightarrow V$ we need additional structure on $V$, which will allow to talk of discrete analogues of differential forms and its integration on compact domains. This is achieved using a cellular complex that uses $V$ as set of vertices (see [4]). In the present work we shall construct a cellular complex using abstract polytopes. For a geometrical description of polytopes, see the appendix.

\section{Definition 3.2}

A nonempty finite subset of points $\beta=\left\{v_{0}, v_{1}, \ldots, v_{k}\right\} \subset \mathbb{R}^{n}$ is called an abstract polytope if none of its points is a convex combination of the remaining ones. An abstract polytope is called an abstract simplex if none of the points is an affine combination of the remaining ones. For abstract simplices with $k+1$ elements, the affine space generated by them has dimension $k$.

For an abstract simplex $\beta$ we call $\operatorname{dim} \beta=\sharp \beta-1$. For arbitrary polytopes we call dimension of the abstract polytope the dimension of the affine space generated by those points, $\operatorname{dim} \beta=\operatorname{dim}\langle\beta\rangle$. Abstract polytopes with dimension 0 are precisely subsets with a single point and are called vertices, abstract polytopes with dimension 1 are precisely subsets with two points and are called edges. For abstract polytopes with dimension higher than 1 , the abstract polytope might not be an abstract simplex. Abstract polytopes in $\mathbb{R}^{n}$ with dimension $n$ are called facets.

For two abstract polytopes $\alpha, \beta \subset \mathbb{R}^{n}$ we say that $\alpha$ is a face of $\beta$ and write $\alpha \prec \beta$ if the convex hull $[\alpha]$ is a face of the convex hull $[\beta]$. For abstract simplices, all its nonempty subsets are faces. 
It is known that the relation of being a face is a symmetric, transitive, and anti-reflexive relation on the abstract polytopes. Moreover, it defines a $T_{0}$ topology:

\section{Definition 3.3}

We say a subset of abstract polytopes $\mathcal{U}$ is closed if for each of its abstract polytopes $\beta \in \mathcal{U}$, the associated faces also belong to $\mathcal{U}$ :

$$
\mathcal{U} \text { closed } \Leftrightarrow(\alpha \prec \beta \in \mathcal{U} \Rightarrow \alpha \in \mathcal{U}) \text {. }
$$

\section{Definition 3.4}

We call $n$-dimensional polytopal complex any finite family $\mathcal{V}$ of polytopes on $\mathbb{R}^{n}$ that is closed and whose maximal elements (with respect to $\prec$ ) are $n$-dimensional abstract polytopes.

$$
\begin{gathered}
\alpha \prec \beta \in \mathcal{V} \Rightarrow \alpha \in \mathcal{V} \\
\alpha \in \mathcal{V}, \operatorname{dim} \alpha \neq n \Rightarrow \exists \beta \in \mathcal{V}, \alpha \prec \beta, \alpha \neq \beta
\end{gathered}
$$

We say the abstract polytopal complex is modelled on a family of points $V \subset \mathbb{R}^{n}$ if all vertices of the family belong to $V$. The subset of $k$-dimensional abstract polytopes of the complex is denoted by $V^{k} \subset \mathcal{V}$.

As $\mathcal{V}$ is closed, we may always assume that it is modelled on $V^{0}$. In the following, we shall write $V$ instead of $V^{0}$ to represent the set of vertices of a polytopal complex $\mathcal{V}$.

It is clear from the definition that, in order to give an $n$-dimensional polytopal complex it suffices to give an arbitrary finite family $V^{n}$ (that we call facets of the complex) representing its $n$-dimensional abstract polytopes. The complex $\mathcal{V}$ is then given as the closure of $V^{n}$, the family of all faces of the facets $\beta \in V^{n}$.

\section{Definition 3.5}

For a given abstract polytope of an abstract polytopal complex $\alpha \in \mathcal{V}$, we call star associated to $\alpha$ the family of abstract polytopes of the complex that have $\alpha$ as face:

$$
\operatorname{Star}_{\alpha}=\{\beta \in \mathcal{V}: \alpha \prec \beta\}
$$

The star can be decomposed using the dimension, as the disjoint union of the $k$-dimensional stars

$$
\operatorname{Star}_{\alpha}^{k}=\operatorname{Star}_{\alpha} \cap V^{k}
$$

Definition 3.6

Let $\mathcal{V}$ be an abstract polytopal complex on a set $V$. We call domain on $\mathcal{V}$ any subset of facets $K \subset V^{n}$. Any such domain generates a corresponding sub-complex $\bar{K} \subset \mathcal{V}$.

An abstract polytpe $\alpha \in \mathcal{V}$ is said to be adherent to the domain $K$ if it is a face of some facet in $K$. The set of adherent polytopes of a domain $K$ shall be denoted by $\bar{K}$.

$$
\alpha \in \bar{K} \Leftrightarrow \exists \beta \in K, \alpha \prec \beta \Leftrightarrow K \cap \operatorname{Star}_{\alpha}^{n} \neq \emptyset
$$

An abstract polytope $\alpha \in \mathcal{V}$ is said to be non-interior to the domain $K$ if it is adherent to $V^{n} \backslash K$. The set of interior polytopes of a domain $K$ shall be denoted by int $K$

$$
\alpha \in \operatorname{int} K \Leftrightarrow \alpha \nprec \beta, \forall \beta \notin K \Leftrightarrow \operatorname{Star}_{\alpha}^{n} \subseteq K
$$

An abstract polytope $\alpha \in \mathcal{V}$ is said to be a frontier polytope of the domain $K$ if it is adherent and non-interior to $K$. The set of frontier polytopes of a domain $K$ shall be denoted by fr $K$

$$
\alpha \in \operatorname{fr} K \Leftrightarrow \alpha \in \bar{K}, \alpha \in \overline{V^{n} \backslash K} \Leftrightarrow K \cap \operatorname{Star}_{\alpha}^{n} \neq \emptyset,\left(V^{n} \backslash K\right) \cap \operatorname{Star}_{\alpha}^{n} \neq \emptyset
$$

Observe that for any domain $K \subset V^{n}$ there holds $\bar{K}=\operatorname{int} K \sqcup$ fr $K$.

Starting from the $n$-dimensional array $V$ with diameter $N$ we shall construct two abstract polytopal complexes: the Cartesian complex, and the Coxeter-Freudenthal-Kuhn simplicial complex. These two abstract complexes represent a discrete model for the space $[1, N]^{n} \subseteq \mathbb{R}^{n}$, described identifying abstract cells with simplices or hypercubes obtained when we split $\mathbb{R}^{n}$ using a particular family of hyperplanes. Fix $r_{1}, \ldots, r_{n}$ as canonical coordinates in $\mathbb{R}^{n}$. 


\section{Proposition 3.1}

Consider the hyperplanes $r_{i}=k$ on $\mathbb{R}^{n}$, one hyperplane for each $i=1 \ldots n$ and each $k \in \mathbb{Z}$. Consider the set $\mathcal{H} \subset \mathbb{R}^{n}$ formed as the union of all these hyperplanes.

The connected components of $\mathbb{R}^{n} \backslash \mathcal{H}$ are the open hypercubes:

$$
C_{v}=\left\{v+\left(\epsilon_{1}, \epsilon_{2}, \ldots, \epsilon_{n}\right): 1>\epsilon_{k}>0, \forall k=1 \ldots n\right\} \subset \mathbb{R}^{n}, \quad v \in \mathbb{Z}^{n}
$$

For any point $r \in \mathbb{R}^{n} \backslash \mathcal{H}$, its connected component is determined by $v=\left(k_{1}, \ldots, k_{n}\right) \in \mathbb{Z}^{n}$ where $k_{i}=\left\lfloor r_{i}\right\rfloor$ (here $\lfloor\cdot\rfloor$ stands for the floor function).

The closure of these hypercubes, $\bar{C}_{v}=\left\{v+\left(\epsilon_{1}, \epsilon_{2}, \ldots, \epsilon_{n}\right): 1 \geq \epsilon_{k} \geq 0, \forall k=1 \ldots n\right\}$, are compact convex sets whose extremal points are:

$$
\operatorname{Ext}\left(\bar{C}_{v}\right)=\left\{v+\left(\epsilon_{1}, \ldots, \epsilon_{n}\right), \epsilon_{i} \in\{0,1\}\right\} \quad\left(v \in \mathbb{Z}^{n}\right)
$$

\section{Definition 3.7}

We call abstract cartesian hypercube determined by an element $v \in \mathbb{Z}^{n}$ the set

$$
\beta(v)=\left\{v+\left(\epsilon_{1}, \ldots, \epsilon_{n}\right), \epsilon_{i} \in\{0,1\}\right\} \subset \mathbb{Z}^{n}
$$

Consider $V$ the $n$-dimensional array with diameter $N$. We call cartesian complex $\mathcal{V}_{\text {cart }}$ on $V$ the abstract polytopal complex generated by all abstract cartesian hypercubes contained in $V$.

Enlarging our family of hyperplanes we may do a partition of these hypercubes into simplices, a partition that appears in different applications (see [37] for example) and had independent origins by Coxeter, Freudenthal and Kuhn:

\section{Proposition 3.2}

Consider the hyperplanes $r_{i}=k$ and the hyperplanes $r_{i_{1}}-r_{i_{2}}=k$ on $\mathbb{R}^{n}$, one hyperplane for each $k \in \mathbb{Z}$ and each integer $i=1 \ldots n$ or pair of integers $1 \leq i_{1}<i_{2} \leq n$. Consider the set $\mathcal{H} \subset \mathbb{R}^{n}$ formed as the union of all these hyperplanes.

The connected components of $\mathbb{R}^{n} \backslash \mathcal{H}$ are the open affine simplices:

$$
K_{v, \sigma}=\left\{v+\epsilon_{1} e_{\sigma(1)}+\ldots+\epsilon_{n} e_{\sigma(n)}, 1>\epsilon_{1}>\epsilon_{2}>\ldots>\epsilon_{n}>0\right\} \quad v \in \mathbb{Z}^{n}, \sigma \in \operatorname{Sym}_{n}
$$

For any point $r \in \mathbb{R}^{n} \backslash \mathcal{H}$, its connected component is determined by $v=\left(k_{1}, \ldots, k_{n}\right) \in \mathbb{Z}^{n}, \quad \sigma=$ $(\sigma(1), \ldots, \sigma(n)) \in \operatorname{Sym}_{n}$ where $k_{i}=\left\lfloor r_{i}\right\rfloor$ and $\sigma$ is the permutation that determines the decreasing order of the fractional components $1>r_{\sigma(1)}-k_{\sigma(1)}>r_{\sigma(2)}-k_{\sigma(2)}>\ldots>r_{\sigma(n)}-k_{\sigma(n)}>0$.

The closure of these affine simplices, $\bar{K}_{v, \sigma}=\left\{v+\epsilon_{1} e_{\sigma(1)}+\ldots+\epsilon_{n} e_{\sigma(n)}, 1 \geq \epsilon_{1} \geq \epsilon_{2} \geq \ldots \geq \epsilon_{n}>0\right\}$, are compact convex sets whose extreme points are:

$$
\operatorname{Ext}\left(\bar{K}_{v, \sigma}\right)=\left\{v_{0}, v_{1}, \ldots, v_{n}\right\}, \quad v_{0}=v, v_{k}=v_{k-1}+e_{\sigma(k)}
$$

That is, all extremal points of the affine simplex $\bar{K}_{v, \sigma}$ are obtained following a path starting at $v$ and sequentially adding all vectors of the canonical basis, following the order determined by $\sigma$.

Proof

Recall that when we remove any hyperplane from $\mathbb{R}^{n}$ this space is disconnected into two convex subsets (open half-spaces). Moreover, intersection of convex subsets is again convex.

Points in $\mathbb{R}^{n} \backslash \mathcal{H}$ are those elements $r \in \mathbb{R}^{n}$ that have noninteger (condition $r_{i} \notin \mathbb{Z}$ ) coordinates $\left(r_{1}, \ldots, r_{n}\right)$, and whose fractional components $f_{i}=r_{i}-\left\lfloor r_{i}\right\rfloor$ are all distinct (condition $r_{i_{1}}-r_{i_{2}} \notin \mathbb{Z}$ ).

Consider the following sets

$$
K_{v, \sigma}=\left\{v+\left(f_{1}, \ldots, f_{n}\right), 1>f_{\sigma(1)}>f_{\sigma(2)}>\ldots>f_{\sigma(n)}>0\right\} \quad v \in \mathbb{Z}^{n}, \sigma=(\sigma(1), \ldots, \sigma(n)) \in \operatorname{Sym}_{n}
$$

Each of these sets is contained in $\mathbb{R}^{n} \backslash \mathcal{H}$, as none of its points have integer coordinates or coincident fractional component on any pair of coordinates. Conversely, any point $r \notin \mathcal{H}$ belongs to some $K_{v, \sigma}$ for some $v=$ 
$\left(k_{1}, \ldots, k_{n}\right)$ and $\sigma \in \mathrm{Sym}_{n}$, it suffices to take $k_{i}=\left\lfloor r_{i}\right\rfloor$ and $\sigma$ the permutation that re-arranges the fractional values $0<f_{i}=r_{i}-k_{i}<1$ in decreasing order.

Moreover, if we call $\epsilon_{i}=f_{\sigma(i)}$ we have:

$$
K_{v, \sigma}=\left\{v+\epsilon_{1} e_{\sigma(1)}+\ldots+\epsilon_{n} e_{\sigma(n)}, 1>\epsilon_{1}>\epsilon_{2}>\ldots>\epsilon_{n}>0\right\} \quad v \in \mathbb{Z}^{n}, \sigma=(\sigma(1), \ldots, \sigma(n)) \in \operatorname{Sym}_{n}
$$

hence the set $K_{v, \sigma}$ is the image of the canonical open simplex $\left\{\left(\epsilon_{1}, \ldots, \epsilon_{n}\right): 1>\epsilon_{1}>\epsilon_{2}>\ldots>\epsilon_{n}>0\right\} \subset \mathbb{R}^{n}$ using the affine isomorphism $\left(\epsilon_{1}, \ldots, \epsilon_{n}\right) \mapsto v+\epsilon_{1} e_{\sigma(1)}+\ldots+\epsilon_{n} e_{\sigma(n)}$. Therefore $K_{v, \sigma}$ are open convex sets, the closure is the image of $\left\{\left(\epsilon_{1}, \ldots, \epsilon_{n}\right): 1>\epsilon_{1}>\epsilon_{2}>\ldots>\epsilon_{n}>0\right\}$ by this isomorphism, and the extreme points are the image of $(0, \ldots, 0),(1,0, \ldots, 0),(1,1,0 \ldots, 0) \ldots$ by this isomorphism. The closure and extreme points are then the ones given in our statement.

As we have a decomposition of $\mathbb{R}^{n} \backslash \mathcal{H}$ as the disjoint union of several open convex (and therefore connected) sets, we conclude that these sets are the connected components in $\mathbb{R}^{n} \backslash \mathcal{H}$.

\section{Definition 3.8}

We call abstract CFK simplex determined by an element $v \in \mathbb{Z}^{n}$ and a permutation $\sigma$, the set

$$
\beta(v, \sigma)=\left\{v_{0}, \ldots, v_{n}\right\}
$$

whose initial element is $v_{0}=v$ and determined by $v_{k+1}=v_{k}+e_{\sigma(k)}$.

Consider $V$ the $n$-dimensional array with diameter $N$. We call CFK complex on $V$ the abstract polytopal complex $\mathcal{V}_{C F K}$ generated by all abstract CFK simplices contained in $V$.

In figure 1 we represent the CFK simplicial partition of a single hypercube, in $\mathbb{R}^{3}$ and in $\mathbb{R}^{2}$ :
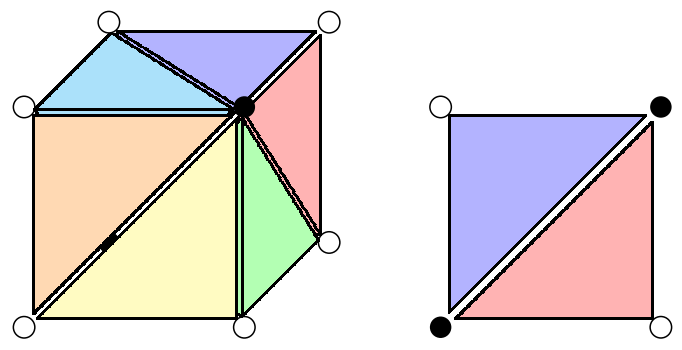

Figure 1. Coxeter-Freudenthal-Kuhn partition in 3D and 2D.

\section{Discrete H-reduced variational principles on the CFK simplicial complex}

On the discrete manifold $V$, seen as a totally disconnected 0-dimensional smooth manifold, all usual objects of bundle theory are available, with the particularity that we are dealing with non-connected manifolds and that certain tangent spaces are the trivial null space. In particular we may consider bundles and principal $G$-bundles on $V$.

\section{Definition 4.1}

We call discrete bundle on a set $V$ any surjective mapping $\pi: Y_{d} \rightarrow V$ whose fibers $\pi^{-1}(v)=Y_{v}$ are smooth manifolds.

We say the discrete bundle $\pi: P_{d} \rightarrow V$ is a discrete principal $G$-bundle if its fibers are the $G$-orbits for some proper, free, smooth action $\lambda: G \rightarrow \operatorname{Aut}\left(P_{d}\right)$ of the Lie group $G$ on $P_{d}$. 
Observe that a discrete bundle is, in particular, a non-connected smooth manifold. As the base manifold is discrete, any tangent vector in the manifold $Y_{d}$ is always vertical with respect to the projector $\pi: Y_{d} \rightarrow V$. We shall call vertical bundle $\pi_{V}: V Y_{d} \rightarrow Y_{d}$ associated to the discrete bundle $Y_{d}$, the vector bundle whose fiber at $y_{v} \in Y_{d}$ is the tangent space at $y_{v}$ of the fiber $Y_{v} \subset Y_{d}$. That is, $\left(V Y_{d}\right)_{y_{v}}=T_{y_{v}} Y_{v}$. This is a smooth vector bundle with a smooth base manifold $Y_{d}$.

Moreover, the restriction of a smooth bundle on an $n$-dimensional manifold $X$ to any particular array with diameter $N$ on this manifold generates a discrete bundle:

\section{Definition 4.2}

Consider $x: V \hookrightarrow X$ an array with diameter $N$ on an $n$-dimensional manifold $X$. For any bundle $\pi: Y \rightarrow X$, we call discrete bundle induced by $Y$ on the array $x$, the bundle $\pi_{x}: Y_{x}=x^{*} Y \rightarrow V$. As a manifold, $Y_{x}$ is the disjoint union of the fibers $Y_{x_{v}}$ and the projector onto $V$ is the determination of the vertex $v \in V$ associated to $\pi(y) \in X$.

Any section $y \in \Gamma(Y)$ of the bundle $\pi: Y \rightarrow X$ naturally induces a section $y_{x}=y \circ x \in \Gamma\left(Y_{x}\right)$, of the discrete bundle induced by the array $x: V \hookrightarrow X$.

Considering that discrete bundles are ordinary bundles where the base manifold is a discrete 0-dimensional manifold, for a discrete principal $G$-bundle we may use most of the main objects in principal bundle theories:

\section{Definition 4.3}

For any discrete principal $G$-bundle $P_{d} \rightarrow V$ and any closed subgroup $H$ of $G$, there exists an induced discrete bundle, the bundle of discrete $H$-structures $\pi_{H S t r}: P_{d} / H=H \operatorname{Str}_{d} \rightarrow V$. This is a smooth manifold with several connected components, and $P_{d}$ itself is a (non-discrete) principal $H$-bundle using $H \operatorname{Str}_{d}$ as (non-discrete) base manifold. We denote $\pi^{H}: P^{H \text { Str }} \rightarrow H \operatorname{Str}_{d}$ this smooth principal $H$-bundle.

\section{Definition 4.4}

The vertical bundles $V^{\pi^{G}} P_{d} \rightarrow P_{d}$ and $V^{\pi^{H}} P^{H \text { Str }} \rightarrow P^{H \text { Str }}$ determine the corresponding quotient bundles, a discrete one $\operatorname{Ad} P_{d}=\left(V P_{d}\right) / G \rightarrow V$ and a smooth one $\operatorname{Ad} P^{H \operatorname{Str}}=\left(V P^{H \mathrm{Str}}\right) / H \rightarrow H \operatorname{Str}_{d}$, adjoint bundles of the principal bundles $P_{d} \rightarrow V$ and $P^{H \text { Str }} \rightarrow H$ Str.

There is a natural inclusion $\operatorname{Ad} P^{H \operatorname{Str}} \subseteq H \operatorname{Str} \times{ }_{V} \operatorname{Ad} P_{d}$.

\section{Definition 4.5}

A discrete principal bundle $\pi: P_{d} \rightarrow V$ determines a corresponding discrete Ehresmann bundle End $P_{d} \rightarrow V \times V$, and for a fixed closed subgroup $H \subseteq G$, also a corresponding smooth Ehresmann bundle End $P^{H \operatorname{Str}} \rightarrow H \operatorname{Str} \times$ $H$ Str.

\section{Remark 4.1}

From [6] we recall that:

- The differential of the gauge difference mapping $\pi^{G}: P_{d} \times P_{d} \rightarrow$ End $P_{d}$ in the first component determines an isomorphism (source trivialisation of $V$ End $P_{d}$ ):

$$
s^{*} \operatorname{Ad} P_{d} \simeq V^{(s, t)} \text { End } P_{d}
$$

where the pull-back of $\operatorname{Ad} P_{d} \rightarrow V$ is performed using $s:$ End $P_{d} \rightarrow V$. In this identification, $\left(\psi_{\alpha}, a_{s(\alpha)}\right) \in$ End $P_{d} \times(s, \pi)$ Ad $P_{d}$ is identified with the tangent vector at $\epsilon=0$ of the trajectory $\exp (-\epsilon a) \cdot \psi_{\alpha}=\psi_{\alpha}$ 。 $\exp (-\epsilon a) \in$ End $P_{\alpha}$.

- The differential of the quotient mapping $\pi^{H}: P_{d} \rightarrow H \operatorname{Str}_{d}$ determines a projector:

$$
\pi_{H \text { Str }}^{*} \operatorname{Ad} P_{d} \rightarrow V H \operatorname{Str}_{d}
$$

with a pull-back performed using $\pi_{H S t r}: H \operatorname{Str}_{d} \rightarrow V$. The kernel of this projector is the sub-bundle Ad $P^{H \text { Str }} \subseteq \pi_{H \text { Str }}^{*} \operatorname{Ad} P_{d}$. In this identification, $\left(q_{v}, a_{v}\right) \in H \operatorname{Str}_{d} \times_{V} \operatorname{Ad} P_{d}$ is identified with the tangent vector at $\epsilon=0$ of the trajectory $q_{v} \exp \epsilon a \in H \operatorname{Str}_{v}$. 
We introduce now the specific facets and edges of our simplicial complex $\mathcal{V}$, which includes additional information not available in the discrete bundle $P_{d} \rightarrow V$. In the following, $V \subset \mathbb{Z}^{n}$ will be the $n$-dimensional array with diameter $N$, where we consider the Coxeter-Freudenthal-Kuhn simplicial complex. For simplicity we shall assume that the lexicographical order is fixed on $V \subset \mathbb{Z}^{n}$ and all subsets of $V$ will be given as (lexicographically) monotone sequences of vertices. Edges will then be given as ordered pairs $\left(v_{0}, v_{1}\right)$, where the vertex $v_{0}$ precedes in the lexicographical order the vertex $v_{1}$. The ordering choice leads to an identification of $V^{1}$ as a particular subset of $V \times V$, and in general an identification of $V^{k}$ as a subset of $V^{\times k}$. Moreover, the projectors $\pi_{i}: V^{\times n} \rightarrow V$, $\pi_{i_{0} i_{1}}=\left(\pi_{i_{0}}, \pi_{i_{1}}\right): V^{\times n} \rightarrow V \times V$ induce corresponding projectors $\pi_{i}: V^{n} \rightarrow V$ and $\pi_{i_{0} i_{1}}: V^{n} \rightarrow V \times V$. For simplicial facets and the case $i_{0}<i_{1}$, we know that $\pi_{i_{0} i_{1}}$ takes values on $V^{1} \subset V \times V$. In a similar way we may define projectors $\pi_{i_{0} \ldots i_{k}}: V^{j} \rightarrow V^{k}$ for the case $0 \leq i_{0}<\ldots<i_{k} \leq j$.

\section{Definition 4.6}

For any discrete bundle $Y_{d} \rightarrow V$, we call extension to $k$-simplices, $Y_{d}^{k} \rightarrow V^{k}$, the restriction of $\left(Y_{d}\right)^{\times k} \rightarrow V^{\times k}$ to the set of $k$-simplices $V^{k} \subset V^{\times k}$.

For any sequence of indices $0 \leq i_{0}<i_{1}<\ldots<i_{k} \leq j$, there exists a projector $\pi_{i_{0} i_{1} \ldots i_{k}}^{Y}: Y_{d}^{j} \rightarrow Y_{d}^{k}$ transforming the sequence $\left(y_{0}, y_{1}, \ldots, y_{j}\right)$ on the simplex $\left(v_{0}, \ldots, v_{j}\right) \in V^{j} \subset V^{\times j}$ into the sequence $\left(y_{i_{0}}, \ldots, y_{i_{k}}\right)$ on the simplex $\pi_{i_{0} \ldots i_{k}}\left(v_{0}, \ldots, v_{j}\right)=\left(v_{i_{0}}, \ldots, v_{i_{k}}\right) \in V^{k} \subset V^{\times k}$. This is a bundle morphism from the bundle $Y_{d}^{j} \rightarrow V^{j}$ to the bundle $Y_{d}^{k} \rightarrow V^{k}$, covering the morphism $\pi_{i_{0} \ldots i_{k}}: V^{j} \rightarrow V^{k}$.

\section{Definition 4.7}

Let $\pi: P_{d} \rightarrow V$ be a discrete bundle and let $(s, t)$ : End $P_{d} \rightarrow V \times V$ be the associated Ehresmann bundle. The fibered product $\left(\text { End } P_{d}\right)^{\times_{s} k-1}=$ End $P_{d} \times{ }_{s}$ End $P_{d} \times{ }_{s} \ldots \times{ }_{s}$ End $P_{d}$ of $k$ copies of $s$ : End $P_{d} \rightarrow V$ has a bundle structure on $V^{\times k}$ using $\left(s, t_{1}, t_{2}, \ldots, t_{k}\right)$ as projector.

For any $k \geq 1$, we call Ehresmann bundle on $k$-simplices, End ${ }^{k} P_{d} \rightarrow V^{k}$, associated to the discrete principal $G$-bundle $P_{d} \rightarrow V$, the restriction of $\left(\text { End } P_{d}\right)^{{ }^{\times} k}$ to the subset $V^{k} \subset V^{\times k}$.

For any sequence of indices $0<i_{1}<\ldots<i_{k} \leq j$ there exists a projector $\pi_{0 i_{1} \ldots i_{k}}^{\mathrm{End}}$ : $\operatorname{End}^{j} P_{d} \rightarrow \operatorname{End}^{k} P_{d}$ transforming the sequence $\left(\psi_{01}, \psi_{02}, \ldots, \psi_{0 j}\right)$ on the simplex $\left(v_{0}, v_{1}, \ldots, v_{j}\right) \in V^{j} \subset V^{\times j}$ into the sequence $\left(\psi_{0 i_{1}}, \psi_{0 i_{2}}, \ldots, \psi_{0 i_{k}}\right)$ on the simplex $\left(v_{0}, v_{i_{1}}, \ldots, v_{i_{k}}\right) \in V^{k} \subset V^{\times k}$. This is a bundle morphism from End ${ }^{j} P_{d} \rightarrow$ $V^{j}$ to the bundle End ${ }^{k} P_{k} \rightarrow V^{k}$ covering the morphism $\pi_{0 i_{1} \ldots i_{k}}: V^{j} \rightarrow V^{k}$.

For any sequence of indices $0<i_{0}<i_{1} \ldots<i_{k} \leq j$, there also exists a projector $\pi_{i_{0} i_{1} \ldots i_{k}}^{\text {End }}$ : End $^{j} P_{d} \rightarrow$ End $^{k} P_{d}$ transforming the sequence $\left(\psi_{01}, \psi_{02}, \ldots, \psi_{0 j}\right)$ on the simplex $\left(v_{0}, v_{1}, \ldots, v_{j}\right) \in V^{j} \subset V^{\times j}$ into the sequence $\left(\psi_{0 i_{0}}^{-1} \psi_{0 i_{1}}, \psi_{0 i_{0}}^{-1} \psi_{0 i_{2}}, \ldots, \psi_{0 i_{0}}^{-1} \psi_{0 i_{k}}\right)$ on the simplex $\left(v_{i_{0}}, v_{i_{1}}, \ldots, v_{i_{k}}\right) \in V^{k} \subset V^{\times k}$. This is a bundle morphism from End ${ }^{j} P_{d} \rightarrow V^{j}$ to the bundle End ${ }^{k} P_{k} \rightarrow V^{k}$ covering the morphism $\pi_{i_{0} i_{1} \ldots i_{k}}: V^{j} \rightarrow V^{k}$.

Particularly relevant will be Ehresmann bundle on edges $\operatorname{End}^{1} P_{d} \rightarrow V^{1}$, which represents the restriction of $(s, t)$ : End $P_{d} \rightarrow V \times V$ to $V^{1} \subset V \times V$. To maintain a uniform notation, the natural projectors $\pi_{0}: V \times V \rightarrow$ $V$ and $\pi_{1}: V \times V \rightarrow V$, when restricted to $V^{1} \subset V \times V$, shall be denoted by $s: V^{1} \rightarrow V$ and $t: V^{1} \rightarrow V$, respectively.

We have just introduced all the relevant elements for the formulation of variational principles on discrete $H$ reduced fields.

\section{Definition 4.8}

For a given discrete principal $G$-bundle $P_{d} \rightarrow V$ and closed subgroup $H \subseteq G$, we call discrete $H$-reduced field, any pair $(q, \psi)$ determined by a section $q \in \Gamma\left(H \operatorname{Str}_{d}\right)$ of the associated bundle of discrete $H$-structures $H \operatorname{Str}_{d}=P_{d} / H \rightarrow V$ on vertices, and another section $\psi \in \Gamma\left(\right.$ End $\left.^{1} P_{d}\right)$ of the associated Ehresmann bundle $(s, t):$ End $^{1} P_{d} \rightarrow V^{1}$ on edges of the CFK complex.

Observe that being all fibers of $H \operatorname{Str}_{d}$ and $\mathrm{End}^{1} P_{d}$ finite-dimensional manifolds, and being both $V$ and $V^{1}$ finite sets, the space $\Gamma\left(H \operatorname{Str}_{d}\right) \times \Gamma\left(\right.$ End $\left.^{1} P_{d}\right)$ of $H$-reduced fields is a manifold with finite (but large) dimension

$$
\Gamma\left(H \operatorname{Str}_{d}\right) \times \Gamma\left(\operatorname{End}^{1} P_{d}\right)=\prod_{v \in V^{0}} H \operatorname{Str}_{v} \times \prod_{\alpha \in V^{1}} \operatorname{End}^{1} P_{\alpha}
$$


Using that the tangent space of a product is the direct sum of tangent spaces of its components, the tangent space at a given point $(q, \psi) \in \Gamma\left(H \operatorname{Str}_{d}\right) \times \Gamma\left(\operatorname{End}^{1} P_{d}\right)$ has a canonical identification:

$$
T_{(q, \psi)}\left(\Gamma\left(H \operatorname{Str}_{d}\right) \times \Gamma\left(\operatorname{End}^{1} P_{d}\right)\right)=\bigoplus_{v \in V^{0}} T_{q_{v}} H \operatorname{Str}_{v} \oplus \bigoplus_{\alpha \in V^{1}} T_{\psi_{\alpha}} \operatorname{End}^{1} P_{\alpha}=\Gamma\left(q^{*} V H \operatorname{Str}_{d}\right) \oplus \Gamma\left(\psi^{*} V \operatorname{End}^{1} P_{d}\right)
$$

\section{Definition 4.9}

We call space of infinitesimal variations of an $H$-reduced discrete field $(q, \psi) \in \Gamma\left(H \operatorname{Str}_{d}\right) \times \Gamma\left(\operatorname{End}^{1} P_{d}\right)$ the vector space $\Gamma\left(q^{*} V H \operatorname{Str}_{d}\right) \oplus \Gamma\left(\psi^{*} V\right.$ End $\left.^{1} P_{d}\right)$. Its elements shall be denoted by $(\delta q, \delta \psi)$, with components $\delta q_{v} \in T_{q_{v}} H \operatorname{Str}_{v}, \delta \psi_{\alpha} \in T_{\psi_{\alpha}}$ End $^{1} P_{\alpha}$ for each $v \in V, \alpha \in V^{1}$.

Moreover, using for each $\psi_{\alpha} \in$ End $P_{d}$ the natural source trivialisation $V_{\psi_{\alpha}}$ End $P_{d} \simeq \operatorname{Ad} P_{s\left(\psi_{\alpha}\right)}$ and for each $q_{v} \in H \operatorname{Str}_{d}$ the natural identification $V_{q_{v}} H \operatorname{Str}_{d} \simeq \operatorname{Ad} P_{\pi_{H S t r} q_{v}} / \operatorname{Ad} P_{q_{v}}^{H \operatorname{Str}}$ we have an identification:

$$
T_{(q, \psi)}\left(\Gamma\left(H \operatorname{Str}_{d}\right) \times \Gamma\left(\operatorname{End}^{1} P_{d}\right)\right) \simeq \Gamma\left(\operatorname{Ad} P_{d} / q^{*} \operatorname{Ad} P^{H \operatorname{Str}}\right) \oplus \Gamma\left(s^{*} \operatorname{Ad} P_{d}\right)
$$

The first component is a section of a vector bundle $\operatorname{Ad} P_{d} \rightarrow V$ on vertices. The second component is a section of a vector bundle $s^{*}$ Ad $P_{d} \rightarrow V^{1}$ on edges.

For the dual space:

$$
T_{(q, \psi)}^{*}\left(\Gamma\left(H \operatorname{Str}_{d}\right) \times \Gamma\left(\operatorname{End}^{1} P_{d}\right)\right) \subset \Gamma\left(\operatorname{Ad}^{*} P_{d}\right) \oplus \Gamma\left(s^{*} \operatorname{Ad}^{*} P_{d}\right)
$$

where the inclusion is as elements whose first component (related to vertices) are linear forms on $\operatorname{Ad} P_{v}$ that vanish on the corresponding subspaces $\operatorname{Ad} P_{q_{v}}^{H \operatorname{Str}} \subseteq \operatorname{Ad} P_{v}$.

\section{Definition 4.10}

We call functional on the space of discrete $H$-reduced fields, any smooth function $\mathbb{L}$ defined on an open subset of the manifold $\Gamma\left(H \operatorname{Str}_{d}\right) \times \Gamma\left(\operatorname{End}^{1} P_{d}\right)$. A discrete $H$-reduced field $(q, \psi)$ is said to be strongly critical for the functional $\mathbb{L}$, if the differential of this function at this point vanishes.

Using the representation of the tangent space as $\Gamma\left(q^{*} V H \operatorname{Str}_{d}\right) \oplus \Gamma\left(\psi^{*} V \operatorname{End}^{1} P_{d}\right)$, the differential of $\mathbb{L}$ at any point is characterized by its components $\mathrm{d}_{(q, \psi)}^{v} \mathbb{L} \in T_{q_{v}}^{*} H \operatorname{Str}_{v}$ (one component for each $v \in V$ ) and $\mathrm{d}_{(q, \psi)}^{\alpha} \mathbb{L} \in$ $T_{\psi_{\alpha}}^{*}$ End $^{1} P_{\alpha}$ (one component for each $\alpha \in V^{1}$ ). It seems, however, more convenient to express them as linear forms on $\operatorname{Ad} P_{d}$ (we get an object not depending on the particular point $(q, \psi)$ ).

Using the identification (6), for any point $(q, \psi) \in \Gamma\left(H \operatorname{Str}_{d}\right) \times \Gamma\left(\operatorname{End}^{1} P_{d}\right)$ there exist unique sections $\partial_{(q, \psi)}^{0} \mathbb{L} \in$ $\Gamma\left(\operatorname{Ad}^{*} P_{d}\right)$ and $\partial_{(q, \psi)}^{1} \mathbb{L} \in \Gamma\left(s^{*} \operatorname{Ad}^{*} P_{d}\right)$ such that $\partial_{(q, \psi)}^{0} \mathbb{L}$ vanishes on $\Gamma\left(q^{*} \operatorname{Ad} P_{d}\right)$, and for which holds:

$$
\left\langle\mathrm{d}_{(q, \psi)} \mathbb{L},\left(\left[a_{v}^{0}\right]_{v \in V},\left(a_{s(\alpha)}^{1}\right)_{\alpha \in V^{1}}\right)\right\rangle=\left\langle\partial_{(q, \psi)}^{0} \mathbb{L}, a^{0}\right\rangle+\left\langle\partial_{(q, \psi)}^{1} \mathbb{L}, a^{1}\right\rangle
$$

at any tangent vector $\left(\left[a_{v}^{0}\right]_{v \in V},\left(a_{s(\alpha)}^{1}\right)_{\alpha \in V^{1}}\right) \in \Gamma\left(\operatorname{Ad} P_{d} / q^{*} \operatorname{Ad} P^{H \operatorname{Str}}\right) \oplus \Gamma\left(s^{*} \operatorname{Ad} P_{d}\right) \simeq T_{(q, \psi)}\left(\Gamma\left(H \operatorname{Str}_{d}\right) \times\right.$ $\Gamma\left(\right.$ End $\left.\left.^{1} P_{d}\right)\right)$, and where $a^{0} \in \Gamma\left(\operatorname{Ad} P_{v}\right)$ stands for any representative in the class $\left[a^{0}\right] \in \Gamma\left(\operatorname{Ad} P_{d} / q^{*} \operatorname{Ad} P^{H \operatorname{Str}}\right)$. The duality product of sections of a bundle and sections of the dual bundle is performed as the summation of duality applied at all of the fibers.

Theorem 4.1

Strongly critical $H$-reduced configurations will be then characterized by:

$$
0=\partial_{(q, \psi)}^{0} \mathbb{L} \in \Gamma\left(\operatorname{Ad}^{*} P_{d}\right), \quad 0=\partial_{(q, \psi)}^{1} \mathbb{L} \in \Gamma\left(s^{*} \operatorname{Ad}^{*} P_{d}\right)
$$

where $\partial_{(q, \psi)}^{0} \mathbb{L} \in \Gamma\left(\operatorname{Ad}^{*} P_{d}\right)$ and $\partial_{(q, \psi)}^{1} \mathbb{L} \in \Gamma\left(s^{*} \operatorname{Ad}^{*} P_{d}\right)$ have components $\partial_{(q, \psi)}^{v} \mathbb{L}$ (with $v \in V$ ) and $\partial_{(q, \psi)}^{\alpha} \mathbb{L}$, (with $\left.\alpha \in V^{1}\right)$ respectively, defined by:

$$
\left(\partial_{(q, \psi)}^{v} \mathbb{L}\right)\left(a_{v}\right)=\mathrm{d}_{(q, \psi)}^{v} \mathbb{L} \in T_{q_{v}}^{*} H \operatorname{Str}_{v} \subseteq \operatorname{Ad}^{*} P_{v}, \quad\left(\partial_{(q, \psi)}^{\alpha} \mathbb{L}\right)=\mathrm{d}_{(q, \psi)}^{\alpha} \mathbb{L} \in T_{\psi_{\alpha}}^{*} \operatorname{End}^{1} P_{\alpha} \simeq \operatorname{Ad}^{*} P_{s(\alpha)}
$$

using the corresponding natural identification and inclusion of dual spaces. 
Proof

Criticality is by definition the annihilation of $\mathrm{d}_{(q, \psi)} \mathbb{L}$, which means the annihilation of all its components. The result is obtained by the natural identifications or immersions of these components in fibers of the dual adjoint bundle.

However we shall not consider arbitrary functionals on the space of discrete $H$-reduced fields, but just action functionals derived from a discrete Lagrangian function. Moreover, we shall not be concerned with strongly critical points of this functional, but only with critical points, with respect to a certain constrained variational principle. We begin with the introduction of action functionals. Recall that given a smooth bundle $Y \rightarrow X$, a Lagrangian density $\mathcal{L}_{\mathrm{Vol}_{X}}$ and a compact domain $K \subset X$, we can define a functional $\mathbb{L}_{K}: y \in \Gamma(Y) \rightarrow \mathbb{L}_{K}(y) \in \mathbb{R}$ on the space of fields $y \in \Gamma(Y)$ using the following steps:

$$
y \in \Gamma(Y) \Rightarrow j y \in \Gamma(J Y) \Rightarrow(\mathcal{L} \circ j y) \operatorname{vol}_{X} \in \Gamma\left(\Lambda^{n} T^{*} X\right) \Rightarrow \int_{K}(\mathcal{L} \circ j y) \operatorname{vol}_{X} \in \mathbb{R}
$$

We want to parallel this process for the case of discrete $H$-reduced fields following a scheme:

$$
\begin{aligned}
(q, \psi) \in \Gamma\left(H \operatorname{Str}_{d}\right) \times \Gamma\left(\operatorname{End}^{1} P_{d}\right) \Rightarrow \operatorname{ex}(q, \psi) \in \Gamma\left(R J P_{d}\right) & \Rightarrow \ell_{d} \circ \operatorname{ex}(q, \psi) \in \Gamma\left(V^{n} \times \mathbb{R}\right) \\
& \Rightarrow \sum_{\beta \in K} \ell_{d} \circ \operatorname{ex}(q, \psi)(\beta) \in \mathbb{R}
\end{aligned}
$$

where $e x: \Gamma\left(H \operatorname{Str}_{d}\right) \times \Gamma\left(\right.$ End $\left.^{1} P_{d}\right) \rightarrow \Gamma\left(R J P_{d}\right)$ should be the discrete operator that extends a given section to a new section, representing the corresponding discrete analogue of a $H$-reduced jet $r j \in \Gamma(J P / H)$.

\section{Definition 4.11}

For any discrete principal $G$-bundle $P_{d} \rightarrow V$ and closed subgroup $H \subseteq G$, we call $R J P_{d}=$ $\pi_{0}^{*} H \operatorname{Str} \times_{V^{n}}$ End $^{n} P_{d} \rightarrow V^{n}$ discrete bundle of $H$-reduced jet configurations associated to $P_{d}$. The bundle $R J P_{d}$ is a discrete bundle on $V^{n}$ whose fiber on any facet $\beta=\left(v_{0}, v_{1}, \ldots, v_{n}\right) \in V^{n} \subset V^{\times n}$ is:

$$
R J P_{\beta}=H \operatorname{Str}_{v_{0}} \times\left(\operatorname{End}^{n} P\right)_{\beta}=H \operatorname{Str}_{v_{0}} \times\left(\operatorname{End}^{1} P\right)_{v_{0} v_{1}} \times \ldots \times\left(\operatorname{End}^{1} P\right)_{v_{0} v_{n}}
$$

In the smooth theory $J P / H=H \operatorname{Str} \times{ }_{X} \mathrm{CP}$, hence an $H$-reduced jet is composed of a point $x \in X$, a single $H$ structure $q_{x} \in H \operatorname{Str}_{x}$, and a principal connection element $\chi_{x} \in \mathrm{CP}_{x}$ at this point, which represents a determination of horizontal lifts $\delta \in T_{x} X \mapsto \chi_{x}(\delta) \in(T P / G)_{x}$ for $n$ independent directions $\delta \in T_{x} X$. For our notion of discrete $H$-reduced jet configuration, the information needed at any fixed facet $\beta$ is a single $H$-structure at the vertex $\pi_{0}(\beta) \in V$, and $n$ fiber-to-fiber endomorphisms for $n$ edges $\pi_{0 i}(\beta) \in V^{1}$.

There are natural projectors defined in $R J P_{d}$. We denote $\pi_{0}^{R J P}: R J P_{d} \rightarrow H \operatorname{Str}_{d}, \pi_{0 i}^{R J P}: R J P_{d} \rightarrow \operatorname{End}^{1} P_{d}$ $(i=1 \ldots n)$ these projectors. They are fibered over $\pi_{0}: V^{n} \rightarrow V$ and $\pi_{0 i}: V^{n} \rightarrow V^{1}$, respectively.

\section{Proposition 4.2}

Consider $s: R J P_{d} \rightarrow V$ the source mapping and $s^{*} \operatorname{Ad} P_{d} \rightarrow R J P_{d}$ the pull-back of the adjoint bundle $\operatorname{Ad} P_{d} \rightarrow$ $V$ to $R J P_{d}$. Consider the sub-bundle $\left(s^{*} \operatorname{Ad} P_{d}\right)^{H \operatorname{Str}} \subset s^{*} \operatorname{Ad} P_{d}$ characterized by:

$$
\left(s^{*} \operatorname{Ad} P_{d}\right)_{r j}^{H \operatorname{Str}}=\operatorname{Ad} P_{q}^{H \operatorname{Str}} \subseteq \operatorname{Ad} P_{v}=\left(s^{*} \operatorname{Ad} P_{d}\right)_{r j}, \quad r j \in R J P_{d}, q=\pi_{0}^{R J P}(r j) \in H \operatorname{Str}_{d}, v=s(r j) \in V
$$

There exists a natural identification of the vertical bundle $V R J P_{d} \rightarrow R J P_{d}$ as:

$$
V R J P_{d} \simeq\left(s^{*} \operatorname{Ad} P_{d}\right) /\left(s^{*} \operatorname{Ad} P_{d}\right)^{H \operatorname{Str}} \oplus\left(s^{*} \operatorname{Ad} P_{d}\right)^{\oplus n-1}
$$

There exists an induced natural immersion of the dual vertical bundle $V^{*} R J P_{d} \rightarrow R J P_{d}$ as sub-bundle of $\left(s^{*} \mathrm{Ad}^{*} P_{d}\right)^{\oplus n} \rightarrow R J P_{d}$ (direct sum of $n+1$ copies of the dual adjoint bundle).

The fiber of this sub-bundle at any $H$-reduced jet configuration $r j=\left(q, \psi_{01}, \ldots, \psi_{0 n}\right) \in R J P_{d}$ with source $s(r j)=v$ is:

$$
V_{r j}^{*} R J P_{d}=\left\{\left(\partial^{0}, \partial^{01}, \ldots, \partial^{0 n}\right):\left\langle\partial^{0}, a\right\rangle=0, \forall a \in \operatorname{Ad} P_{q}^{H \operatorname{Str}} \subset \operatorname{Ad} P_{v}\right\} \subseteq\left(\operatorname{Ad}^{*} P_{v}\right)^{\oplus n}
$$


Proof

Using the definition of $R J P_{d}$ as a fibered product, the projectors to each of its components determine an isomorphism:

$$
V_{r j} R J P_{d}=V_{q_{0}} H \operatorname{Str}_{d} \oplus \bigoplus_{i=1 \ldots n} V_{\psi_{0 i}} \operatorname{End}^{1} P_{d}, \quad q_{0}=\pi_{0}^{R J P}(r j), \psi_{0 i}=\pi_{0 i}^{R J P}(r j)
$$

using now the natural identification $V_{q} H \operatorname{Str}_{d} \simeq \operatorname{Ad} P_{\pi(q)} / \operatorname{Ad} P_{q}^{H S \operatorname{Str}}$ and the source trivialisation $V_{\psi}$ End $^{1} P_{d} \simeq$ Ad $P_{s(\psi)}$, one obtains the result for the vertical bundle.

In the dual vertical bundle, it suffices to observe that the quotient morphism $E \rightarrow E / F$ of any vector bundle $E$ by some sub-bundle $F$ induces a corresponding immersion of dual bundles $(E / F)^{*} \hookrightarrow E^{*}$, identifying $(E / F)^{*}$ as the sub-bundle of linear forms on $E$ that vanish when applied to $F \subset E$.

Definition 4.12

For any smooth function $\ell: R J P_{d} \rightarrow \mathbb{R}$, the $n+1$ components in $\mathrm{Ad}^{*} P_{s(r j)}$ determined by its differential at a given point $r j \in R J P_{d}$ are denoted by $\partial_{r j}^{0} \ell, \partial_{r j}^{01} \ell, \ldots, \partial_{r j}^{0 n} \ell$.

Remark 4.2

Observe that every discrete $H$-reduced field $(q, \psi) \in \Gamma\left(H \operatorname{Str}_{d}\right) \times \Gamma\left(\right.$ End $\left.^{1} P_{d}\right)$ determines a corresponding discrete $H$-reduced jet $e x(q, \psi) \in \Gamma\left(R J P_{d}\right)$, namely a section $\beta \in V^{n} \mapsto e x_{\beta}(q, \psi) \in R J P_{d}$ defined by:

$$
\beta=\left(v_{0}, \ldots, v_{n}\right) \in V^{n} \Rightarrow e x_{\beta}(q, \psi)=\left(q_{v_{0}}, \psi_{v_{0} v_{1}}, \ldots, \psi_{v_{0} v_{n}}\right)
$$

Observe that $\Gamma\left(R J P_{d}\right)$ is a direct product $\prod_{\beta \in V^{n}}\left(H \operatorname{Str}_{\pi_{0}(\beta)} \times \prod_{i=1 \ldots n}\right.$ End $\left.^{1} P_{\pi_{0 i}(\beta)}\right)$ and has then a smooth manifold structure. We may denote by $r j_{\beta}^{0} \in H \operatorname{Str}_{\pi_{0}(\beta)}$ and by $r j_{\beta}^{0 i} \in \operatorname{End}^{1} P_{\pi_{0 i}(\beta)}$ the corresponding components of each section $r j \in \Gamma\left(R J P_{d}\right)$. Consequently, the tangent space of $\Gamma\left(R J P_{d}\right)$ at any $r j \in \Gamma\left(R J P_{d}\right)$ is a direct sum:

$$
T_{r j}\left(\Gamma\left(R J P_{d}\right)\right)=\bigoplus_{\beta \in V^{n}}\left(T_{r j_{\beta}^{0}} H \operatorname{Str}_{\pi_{0}(\beta)} \oplus \bigoplus_{i=1 \ldots n} T_{r j_{\beta}^{0 i}} \operatorname{End}^{1} P_{\pi_{0 i}(\beta)}\right)=\Gamma\left(r j^{*} V R J P_{d}\right)
$$

\section{Proposition 4.3}

The mapping $e x: \Gamma\left(H \operatorname{Str}_{d}\right) \times \Gamma\left(\operatorname{End}^{1} P_{d}\right) \rightarrow \Gamma\left(R J P_{d}\right)$ is smooth. The differential at any given $(q, \psi)$ is described in components $\left(\delta q_{v}, \delta \psi_{\alpha}\right) \mapsto\left(\delta r j_{\beta}^{0}, \delta r j_{\beta}^{0 i}\right)$ as:

$$
\delta r j_{\beta}^{0}=\delta q_{\pi_{0}(\beta)}, \quad \delta r j_{\beta}^{0 i}=\delta \psi_{\pi_{0 i}(\beta)}, \quad \forall \beta \in V^{n}, i=1 \ldots n
$$

Proof

The mapping $e x$ is merely a re-ordering (with possible repetitions) of the several components $q_{v}, \psi_{\alpha}$ in $(q, \psi)$ to give the different components of $e x(q, \psi)$. It can be described as:

$$
r j_{\beta}^{0}=q_{\pi_{0}(\beta)}, \quad r j_{\beta}^{0 i}=\psi_{\pi_{0 i}(\beta)}, \quad \forall \beta \in V^{n}, i=1 \ldots n
$$

As a consequence, it is a smooth mapping, and the induced mapping on the tangent spaces is the one given in the statement.

We may observe from (7) that sections $r j \in \Gamma\left(R J P_{d}\right)$ that are obtained in the form $e x(q, \psi)$ must always satisfy the relations:

$$
\begin{aligned}
r j_{\beta_{1}}^{0 i_{1}} & =r j_{\beta_{2}}^{0 i_{2}}, \quad \forall \beta_{1}, \beta_{2} \in V^{n}, \quad i_{1}, i_{2}=1 \ldots n \text { such that } \pi_{0 i_{1}}\left(\beta_{1}\right)=\pi_{0 i_{2}}\left(\beta_{2}\right) \in V^{1} \\
r j_{\beta}^{0} & =r j_{\bar{\beta}}^{0}, \quad \forall \beta, \bar{\beta} \in V^{n}, \text { such that } \pi_{0}(\beta)=\pi_{0}(\bar{\beta}) \in V
\end{aligned}
$$

In the same manner as not all sections of a jet bundle are a jet extension of a field, also not all sections of the discrete bundle of $H$-reduced jet configurations are a reduced jet extension of some discrete $H$-reduced field $(q, \psi)$. 


\section{Definition 4.13}

We call discrete $H$-reduced Lagrangian function, any locally defined function $\ell_{d}: R J P_{d} \rightarrow \mathbb{R}$, whose domain is an open sub-bundle $\widetilde{R J P}_{d} \subset R J P_{d} \rightarrow V^{n}$ with non-empty fibers. Elements in the domain shall be called regular $H$-reduced jet configurations.

As $R J P_{d}$ is a discrete union of its fibers $R J P_{\beta}$, any discrete $H$-reduced Lagrangian function $\ell_{d}$ can be seen as a family of smooth functions $\left\{\ell_{\beta}\right\}_{\beta \in V^{n}}$, each of them defined on a nonempty open subset of a fiber $\widetilde{R J P}_{\beta} \subset R J P_{\beta}$. Following Definition 4.12, the components of the differential $\mathrm{d} \ell_{\beta}$ at a certain point $r j \in R J P_{\beta}$ with $s(r j)=\pi_{0}(\beta)=v \in V$, using the natural immersion $V_{r j}^{*} R J P_{d} \subseteq\left(\operatorname{Ad}^{*} P_{v}\right)^{\oplus n}$, shall be denoted by $\partial_{r j}^{0} \ell_{\beta}$, $\partial_{r j}^{01} \ell_{\beta}, \ldots, \partial_{r j}^{0 n} \ell_{\beta} \in \mathrm{Ad}^{*} P_{v}$.

\section{Remark 4.3}

In the case that the discrete principal bundle $P_{d} \rightarrow V$ is the restriction $P_{d}=x^{*} P \subset P$ of some smooth bundle $P \rightarrow$ $X$ to some array $x: V \rightarrow X$, we have $H \operatorname{Str}_{d}=P_{d} / H \subset P / H=H \operatorname{Str}$ and End ${ }^{1} P_{d} \subset$ End $P_{d} \subset$ End $P$, therefore End $^{n} P_{d} \subset(\text { End } P)^{\times_{s} n-1}$. Hence, $R J P_{d} \subset H \operatorname{Str} \times_{X}(\text { End } P)^{\times_{s} n-1}$ and an $H$-reduced Lagrangian function can be obtained considering a single smooth function $\ell: H \operatorname{Str} \times{ }_{X}(\text { End } P)^{\times}{ }_{s} n-1 \rightarrow \mathbb{R}$ and its corresponding restriction to a finite family of fibers $\left(x\left(v_{0}\right), \ldots, x\left(v_{n}\right)\right) \in X^{\times n}$, for all choices $\left(v_{0}, \ldots, v_{n}\right) \in V^{n} \subset V^{\times n}$. In the case of a trivial principal $G$-bundle this class of $H$-reduced Lagrangian functions can be written in the form $\ell\left(x_{0}, \ldots, x_{n}, H g, g_{01}, \ldots, g_{0 n}\right)$ for some smooth function on $X^{\times n} \times G / H \times G^{\times n-1}$, or in the simplest case $\ell$ has an expression $\ell\left(H g, g_{01}, \ldots, g_{0 n}\right)$, smooth function defined on a manifold $G / H \times G \times \ldots \times G$.

\section{Definition 4.14}

Consider a discrete $H$-reduced Lagrangian function $\ell_{d}: R J P_{d} \rightarrow \mathbb{R}$, defined on a certain open domain $\widetilde{R J P}_{d} \subset$ $R J P_{d}$ (of regular $H$-reduced jet configurations). We say a discrete $H$-reduced field $(q, \psi)$ belongs to the regular domain if its $H$-reduced jet extension $e x(q, \psi) \in \Gamma\left(R J P_{d}\right)$ takes values in the regular domain $\widetilde{R J P}_{d}$. In this case, we say that the discrete action functional $\mathbb{L}_{d}$ determined by $\ell_{d}$ takes at $(q, \psi)$ the following value:

$$
\mathbb{L}_{d}(q, \psi)=\sum_{\beta \in V^{n}} \ell_{\beta}\left(e x_{\beta}(q, \psi)\right)
$$

We say an $H$-reduced field $(q, \psi)$ is strongly critical for the $H$-reduced Lagrangian function $\ell_{d}$, if it is strongly critical for the associated functional $\mathbb{L}_{d}$.

Observe that, in the same way that there might not exist globally defined smooth sections of a bundle, in the discrete case, where the existence of globally defined sections is guaranteed, the condition that the extension of the section to facets should lie on a certain domain of regular configurations might be too strong. For certain choices, the domain of regular configurations could be so small that the discrete action functional cannot be computed on any global section.

To compute strongly critical points for some $H$-reduced Lagrangian function $\ell_{d}$, one must compute the derivative of the locally defined function $\mathbb{L}_{d}: \Gamma\left(H \operatorname{Str}_{d}\right) \times \Gamma\left(\operatorname{End}^{1} P_{d}\right) \rightarrow \mathbb{R}$ at points $(q, \psi) \in \Gamma\left(H \operatorname{Str}_{d}\right) \times \Gamma\left(\operatorname{End}^{1} P_{d}\right)$. Using the chain rule, this is performed computing the differential of each function $\ell_{\beta}$, for each facet $\beta \in V^{n}$, and the differential of $e x$, given in proposition (4.3), leading to:

\section{Proposition 4.4}

Consider an $H$-reduced field $(q, \psi) \in \Gamma\left(H \operatorname{Str}_{d}\right) \times \Gamma\left(\operatorname{End}^{1} P_{d}\right)$ and the induced $H$-reduced jet field $e x(q, \psi)=$ $r j \in \Gamma\left(R J P_{d}\right)$. The differential $\mathrm{d}_{(q, \psi)} \mathbb{L} \in \Gamma\left(q^{*} V^{*} H \operatorname{Str}_{d}\right) \oplus \Gamma\left(\psi^{*} V^{*} \operatorname{End}^{1} P_{d}\right)$ at $(q, \psi)$, of the discrete action functional $\mathbb{L}_{d}$, associated to some discrete $H$-reduced Lagrangian density $\ell_{d}=\left(\ell_{\beta}\right)_{\beta \in V^{n}}$, has components depending on $\ell_{\beta}$ through:

$$
\partial_{(q, \psi)}^{v} \mathbb{L}=\sum_{\pi_{0}(\beta)=v} \partial_{r j_{\beta}}^{0} \ell_{\beta} \in \operatorname{Ad}^{*} P_{v}, \quad \partial_{(q, \psi)}^{\alpha} \mathbb{L}=\sum_{i=1}^{n} \sum_{\pi_{0 i}(\beta)=\alpha} \partial_{r j_{\beta}}^{0 i} \ell_{\beta} \in \operatorname{Ad}^{*} P_{v}
$$

Corollary 4.1

When the action functional is defined by a discrete $H$-reduced Lagrangian function $\ell: R J P_{d} \rightarrow \mathbb{R}$, strongly critical 
discrete $H$-reduced fields will be characterized by:

$$
0=\sum_{\pi_{0}(\beta)=v} \partial_{r j_{\beta}}^{0} \ell_{\beta}, \quad 0=\sum_{i=1}^{n} \sum_{\pi_{0 i}(\beta)=\alpha} \partial_{r j_{\beta}}^{0 i} \ell_{\beta}
$$

However we shall not seek for $H$-reduced fields that ate strongly critical. The discrete variational principle will consider only a certain subset of discrete $H$-reduced fields (admissible fields) and a certain subspace of (admissible) infinitesimal variations for these fields.

\section{Constrained variational principles on discrete H-reduced fields}

In a similar way as was done in the smooth theory of Euler-Poincare reduction, we shall look for global $H$-reduced fields, that are critical points for the discrete action functional, but only with respect to some restricted set of variations.

\section{Definition 5.1}

We say that $(q, \psi) \in \Gamma\left(H \operatorname{Str}_{d}\right) \times \Gamma\left(\operatorname{End}^{1} P_{d}\right)$ is an admissible $H$-reduced field if for any edge $\left(v_{0}, v_{1}\right) \in V^{1} \subset$ $V \times V$ there holds $q_{v_{0}} \psi_{v_{0} v_{1}}=q_{v_{1}}$ and for any 2-simplex $\left(v_{0}, v_{1}, v_{2}\right) \in V^{2} \subset V^{\times 2}$ there holds $\psi_{v_{0} v_{1}} \psi_{v_{1} v_{2}}=\psi_{v_{0} v_{2}}$.

This definition recovers the notion of parallel $H$-structure and flat connection assumed for admissible $H$ reduced fields in the smooth theory. In order to determine a constrained variational principle on discrete $H$ reduced fields, we still need to fix compatible subspaces of admissible infinitesimal variations, for each admissible $(q, \psi) \in \Gamma\left(H \operatorname{Str}_{d}\right) \times \Gamma\left(\operatorname{End}^{1} P_{d}\right)$.

\section{Remark 5.1}

For any admissible discrete $H$-reduced field $(q, \psi)$ and for any $a \in \Gamma\left(\operatorname{Ad} P_{d}\right)$, the family of discrete $H$-reduced fields $(q(\epsilon), \psi(\epsilon))$ described by $q_{v}(\epsilon)=q_{v} \exp \epsilon a, \psi_{\alpha}(\epsilon)=\left(\exp -\epsilon a_{s(\alpha)}\right) \psi_{\alpha} \exp \epsilon a_{t(\alpha)}$ are also admissible.

The tangent vector to the curve $(q(\epsilon), \psi(\epsilon))$ in the source-trivialisation (5) has components:

$$
\delta q_{v}=\left[a_{v}\right], \quad \delta \psi_{\alpha}=a_{s(\alpha)}-\operatorname{Ad}_{\psi_{\alpha}^{-1}} a_{t(\alpha)} \quad\left(v \in V, \alpha \in V^{1}\right)
$$

\section{Definition 5.2}

For any admissible discrete $H$-reduced field $(q, \psi) \in \Gamma(H \mathrm{Str}) \times \Gamma\left(\operatorname{End}^{1} P\right)$, we call space of admissible variations $\mathcal{A} \mathcal{V}_{(q, \psi)}$, the image of the linear operator:

$$
\mathcal{P}_{(q, \psi)}: a \in \Gamma\left(\operatorname{Ad} P_{d}\right) \mapsto\left(\left(\left[a_{v}\right]\right)_{v \in V},\left(a_{s(\alpha)}-\operatorname{Ad}_{\psi_{\alpha}^{-1}} a_{t(\alpha)}\right)_{\alpha \in V^{1}}\right) \in \Gamma\left(q^{*} V H \operatorname{Str}_{d}\right) \oplus \Gamma\left(\psi^{*} V \operatorname{End}^{1} P\right)
$$

We are in situation to define the constrained variational problem in discrete $H$-reduced fields. It is given as:

- An abstract (ordered) simplicial complex $\mathcal{V}$ modelled on a finite set of vertices $V^{0}=V$.

- A discrete principal $G$-bundle $P_{d} \rightarrow V$, its associated discrete Ehresmann groupoid on edges $\pi_{\text {End }}:$ End $^{1} P_{d} \rightarrow V^{1}$ and discrete adjoint bundle $\pi_{\mathrm{Ad}}: \operatorname{Ad} P_{d} \rightarrow V$ on vertices.

- A closed subgroup $H \subseteq G$ determining the associated discrete $H$-structure bundle $\pi_{H \mathrm{Str}}: H \operatorname{Str}_{d} \rightarrow V$, principal $H$-bundle $\pi^{H}: P^{H \mathrm{Str}} \rightarrow H \mathrm{Str}_{d}$ and the bundle of $H$-reduced jet configurations $R J P_{d} \rightarrow V^{n}$.

- A smooth, locally defined, function (discrete $H$-reduced Lagrangian) $\ell_{d}: R J P_{d} \rightarrow \mathbb{R}$.

In this situation we may define:

- The manifold of discrete $H$-reduced fields $\Gamma\left(H \operatorname{Str}_{d}\right) \times \Gamma\left(\operatorname{End}^{1} P_{d}\right)$, and the subset of admissible fields, characterized for having a flat discrete connection that can be reduced to the $H$-structure (we may also say that the $H$-structure is parallel).

- For each admissible $H$-reduced field $(q, \psi)$, a corresponding space of admissible infinitesimal variations $\mathcal{A} \mathcal{V}_{(q, \psi)} \subset \Gamma\left(q^{*} V H \operatorname{Str}_{d}\right) \oplus \Gamma\left(\psi^{*} V \operatorname{End}^{1} P_{d}\right)$. 
- An action functional $\mathbb{L}_{d}: \Gamma\left(H \operatorname{Str}_{d}\right) \times \Gamma\left(\right.$ End $\left.^{1} P_{d}\right) \rightarrow \mathbb{R}$ determined by the $H$-reduced Lagrangian function $\ell_{d}$.

\section{Definition 5.3}

Se say an admissible discrete $H$-reduced field $(q, \psi) \in \Gamma\left(H \operatorname{Str}_{d}\right) \times \Gamma\left(\operatorname{End}^{1} P_{d}\right)$ is critical for the constrained variational problem determined by the discrete $H$-reduced Lagrangian function $\ell_{d}$, if the differential $\mathrm{d}_{(q, \psi)} \mathbb{L}_{d}$ of the action functional vanishes on the subspace of admissible infinitesimal variations $\mathcal{A} \mathcal{V}_{(q, \psi)}$.

Obviously, in the case that there exists a strongly critical, admissible $H$-reduced field $(q, \psi)$ for $\ell_{d}$, it will be critical for the constrained variational problem determined by $\ell_{d}$.

Theorem 5.1

An admissible discrete $H$-reduced field $(q, \psi) \in \Gamma\left(H \operatorname{Str}_{d}\right) \times \Gamma\left(\operatorname{End}^{1} P_{d}\right)$ is critical for the constrained variational problem determined by $\ell_{d}$ if and only if the discrete Euler-Poincaré equations $0=\mathcal{E P}(q, \psi)$ hold, where $\mathcal{E P}(q, \psi) \in \Gamma\left(\operatorname{Ad}^{*} P_{d}\right)$ is described by:

$$
\mathcal{E P}_{v}(q, \psi)=\sum_{\pi_{0}(\beta)=v} \partial_{r j_{\beta}}^{0} \ell_{\beta}+\sum_{i=1}^{n} \sum_{\pi_{0}(\beta)=v} \partial_{r j_{\beta}}^{0 i} \ell_{\beta}-\sum_{i=1}^{n} \sum_{\pi_{i}(\beta)=v} \operatorname{Ad}_{\psi_{\pi_{0 i}(\beta)}}^{*} \partial_{r j_{\beta}}^{0 i} \ell_{\beta}, \quad r j=e x(q, \psi) \in \Gamma\left(R J P_{d}\right)
$$

where $\operatorname{Ad}_{\psi}^{*}: \operatorname{Ad}^{*} P_{s(\psi)} \rightarrow \operatorname{Ad}^{*} P_{t(\psi)}$ stands for the linear morphism induced in the dual adjoint spaces by the morphism $\operatorname{Ad}_{\psi^{-1}}: \operatorname{Ad} P_{t(\psi)} \rightarrow \operatorname{Ad} P_{s(\psi)}$.

Proof

For a fixed $u \in V$, and considering the section $a \in \Gamma\left(\operatorname{Ad} P_{d}\right)$ that takes value $a_{u} \in \operatorname{Ad} P_{u}$ at the vertex $u$ and vanishes everywhere else we have:

$$
\begin{aligned}
\left\langle\mathrm{d}_{(q, \psi)} \mathbb{L},\right. & \left.\mathcal{P}_{(q, \psi)}(a)\right\rangle=\left\langle\partial_{(q, \psi)}^{0} \mathbb{L},\left[a_{v}\right]_{v \in V}\right\rangle+\left\langle\partial_{(q, \psi)}^{1} \mathbb{L},\left(a_{s(\alpha)}-\operatorname{Ad}_{\psi_{\alpha}^{-1}} a_{t(\alpha)}\right)_{\alpha \in V^{1}}\right\rangle= \\
& =\left\langle\partial_{(q, \psi)}^{u} \mathbb{L}, a_{u}\right\rangle+\sum_{s(\alpha)=u}\left\langle\partial_{(q, \psi)}^{\alpha} \mathbb{L}, a_{u}\right\rangle-\sum_{t(\alpha)=u}\left\langle\partial_{(q, \psi)}^{\alpha} \mathbb{L}, \operatorname{Ad}_{\psi_{\alpha}^{-1}} a_{u}\right\rangle= \\
& =\left\langle\partial_{(q, \psi)}^{u} \mathbb{L}+\sum_{s(\alpha)=u} \partial_{(q, \psi)}^{\alpha} \mathbb{L}-\sum_{t(\alpha)=u} \operatorname{Ad}_{\psi_{\alpha}}^{*} \partial_{(q, \psi)}^{\alpha} \mathbb{L}, a_{u}\right\rangle
\end{aligned}
$$

here $\mathrm{Ad}_{\psi}^{*}$ stands for the linear mapping $\operatorname{Ad}^{*} P_{s(\psi)} \rightarrow \operatorname{Ad}^{*} P_{t(\psi)}$ induced in the dual spaces by $\operatorname{Ad}_{\psi^{-1}}: \operatorname{Ad}_{t(\psi)} \rightarrow$ Ad $P_{s(\psi)}$.

As any section $a \in \Gamma\left(\operatorname{Ad} P_{d}\right)$ is a finite sum of sections of the given form, we conclude that criticality is equivalent to the vanishing of $\mathcal{E P}(q, \psi) \in \Gamma\left(\operatorname{Ad}^{*} P_{d}\right)$ defined by:

$$
\mathcal{E} \mathcal{P}_{u}(q, \psi)=\partial_{(q, \psi)}^{u} \mathbb{L}+\sum_{s(\alpha)=u} \partial_{(q, \psi)}^{\alpha} \mathbb{L}-\sum_{t(\alpha)=u} \operatorname{Ad}_{\psi_{\alpha}}^{*} \partial_{(q, \psi)}^{\alpha} \mathbb{L}
$$

To express this element in terms of the discrete $H$-reduced Lagrangian function $\ell_{d}: R J P_{d} \rightarrow \mathbb{R}$, we may use (8) to obtain:

$$
\mathcal{E} \mathcal{P}_{u}(q, \psi)=\sum_{\pi_{0}(\beta)=u} \partial_{r j_{\beta}}^{0} \ell_{\beta}+\sum_{i=1}^{n} \sum_{\pi_{0}(\beta)=u} \partial_{r j_{\beta}}^{0 i} \ell_{\beta}-\sum_{i=1}^{n} \sum_{\pi_{i}(\beta)=u} \operatorname{Ad}_{\psi_{\pi_{0 i}(\beta)}}^{*} \partial_{r j_{\beta}}^{0 i} \ell_{\beta}
$$

\section{Integrators for discrete Euler-Poincaré equations}

Observe the structure of discrete Euler-Poincaré equations (9). Suppose we have an admissible discrete $H$-reduced field $(q, \psi) \in \Gamma\left(H \operatorname{Str}_{d}\right) \times \Gamma\left(\operatorname{End}^{1} P_{d}\right)$, that is critical for a certain discrete Lagrangian function $\ell_{d}$. 
Let us decompose the space $\mathbb{Z}^{n}$ into slices $S_{c}$ defined by equations $k_{1}+k_{2}+\ldots+k_{n}=c$. Consider a vertex $u \in \mathbb{Z}^{n}$ in a given slice $S_{c+n}$. Assume that all values $q_{w}, \psi_{v_{0} w}$ are known for vertices $w$ in the region $k_{1}+\ldots+k_{n}<c+n$. What can be said about the values $q_{u}$ and $\psi_{v_{0} u}$ ?

Taking Euler-Poincaré equations at $v=u-(1, \ldots, 1)$ (hence $v \in S_{c}$ ), we observe that the equation $0=$ $\mathcal{E P}_{v}(q, \psi)$ only depends on $r j_{\beta}$ when $\pi_{i}(\beta)=v$ for some $i$. This implies $\pi_{0}(\beta) \in S_{c-i}$, and $r j_{\beta}$ only depends in configurations $q_{w}, \psi_{v_{0}} w$ with $w$ in the region $k_{1}+\ldots+k_{n}<c+n$, plus the particular configuration $\psi_{v u}$ (that appears in components $r j_{\beta}$ when $\pi_{0}(\beta)=v$ ).

If we decompose Euler-Poincaré equations at $v$ into components that depends on $r j_{\beta}$ for $\pi_{0}(\beta)=v$ and another one that depends on $r j_{\beta}$ for $\pi_{i}(\beta)=v, i=1 \ldots n,(9)$ is equivalent to:

$$
\overbrace{\sum_{\pi_{0}(\beta)=v} \partial_{r j_{\beta}}^{0} \ell_{\beta}+\sum_{i=1}^{n} \sum_{\pi_{0}(\beta)=v} \partial_{r j_{\beta}}^{0 i} \ell_{\beta}}^{E P 1}=\overbrace{\sum_{i=1}^{n} \sum_{\pi_{i}(\beta)=v} \operatorname{Ad}_{\psi_{\pi_{0 i}(\beta)}}^{*} \partial_{r j_{\beta}}^{0 i} \ell_{\beta}}^{E P 2}
$$

When $q_{w}, \psi_{v_{0} w}$ are known in the region $k_{1}+\ldots+k_{n}<c+n$, the right hand side in the equations is known, and most components on the left hand side are also known, except for the particular component $\psi_{v u}$ with $u=v+(1, \ldots, 1)$. If $\operatorname{dim} G=m$ (and consequently $\operatorname{dim} \operatorname{Ad}^{*} P_{v}=m, \operatorname{dim} \operatorname{End}^{1} P_{u v}=m$ ) we have a system of $m$ equations with $\psi_{v u}$ as unknown that taking into account the dimensions, in some regular cases, will determine a unique solution.

For any subset of indices $S \subset[n]=\{1,2, \ldots, n\}$, denote $e_{S}$ the vector with component 1 for any index in $S$ and component 0 for any index not belonging to $S$.

$$
e_{S}=\left(c_{1}, \ldots, c_{n}\right), c_{i}=1 \text { if } i \in S, c_{i}=0 \text { if } i \notin S
$$

For the CFK simplicial complex we easily observe for a fixed vertex:

\section{Lemma 6.1}

For all facets with $\pi_{0}(\beta)=v$, the corresponding family of edges $\pi_{0 i}(\beta)$ are all edges $\alpha(v, S)=\left(v, v+e_{S}\right)$ with $\emptyset \neq S \subset[n]=\{1, \ldots, n\}$.

\section{Definition 6.1}

We call bundle of discrete $H$-reduced forward configurations, Forw $_{d}^{H} \rightarrow V$, the bundle whose fiber on a vertex $v \in V$ is the following

$$
\operatorname{Forw}_{v}^{H}=H \operatorname{Str}_{v} \times \prod_{\emptyset \neq S \subseteq[n]} \operatorname{End}^{1} P_{\alpha(v, S)}
$$

We call bundle of discrete $H$-reduced backward configurations, Back $_{d}^{H} \rightarrow V$, the product bundle of all $H$-reduced jet configurations at facets containing $v$ but not with source $v$ :

$$
\operatorname{Back}_{v}^{H}=\prod_{i=1}^{n} \prod_{\pi_{i}(\beta)=v} R J P_{\beta}
$$

We observe that the decomposition of discrete Euler-Poincaré equations lead to a component $E P_{1}$ defined on Forw ${ }_{d}^{H}$ and a second one $E P_{2}$ defined on $\operatorname{Back}_{d}^{H}$.

\section{Definition 6.2}

We call Legendre transformation associated to a discrete $H$-reduced Lagrangian $\ell_{d}$, the mapping Leg: Forw $_{d}^{H} \rightarrow$ $\operatorname{Ad}^{*} P_{d}$ defined on each fiber by:

$$
\operatorname{Leg}_{v}:\left(q_{v},\left(\psi_{\alpha(v, S)}\right)_{\emptyset \neq S \subseteq[n]}\right) \in \operatorname{Forw}_{v}^{H} \mapsto \sum_{\pi_{0}(\beta)=v} \partial_{r j_{\beta}}^{0} \ell_{\beta}+\sum_{i=1}^{n} \sum_{\pi_{0}(\beta)=v} \partial_{r j_{\beta}}^{0 i} \ell_{\beta} \in \operatorname{Ad}^{*} P_{v}
$$

where $r j_{\beta}=\left(q_{v},\left(\psi_{\alpha}\right)_{\alpha \prec \beta}\right)$. 
We call momentum mapping associated to $\ell_{d}$, the mapping Mom: $\mathrm{Back}^{H} \rightarrow \mathrm{Ad}^{*} P$ defined on each fiber by:

$$
\operatorname{Mom}_{v}:\left(r j_{\beta}\right)_{s(\beta) \neq v \prec \beta} \in \operatorname{Back}_{v}^{H} \mapsto \sum_{i=1}^{n} \sum_{\pi_{i}(\beta)=v} \operatorname{Ad}_{\psi_{\pi_{0 i}(\beta)}}^{*} \partial_{r j_{\beta}}^{0 i} \ell_{\beta} \in \operatorname{Ad}^{*} P_{v}
$$

The central step in solving discrete Euler-Poincaré equations lies in the determination of a single component $\psi_{\Delta v}$, for some edge $\Delta v=(v, v+(1, \ldots, 1)) \in V^{1}$, using the remaining available configurations.

Lemma 6.2

Consider the mapping $\Delta: v \in V \mapsto(v, v+(1, \ldots, 1)) \in V^{1}$. Consider the bundle $\Delta^{*}$ End $^{1} P_{d} \rightarrow V$ (whose fiber on a vertex $v$ is precisely $\operatorname{End}^{1} P_{\Delta(v)}$. The bundle of discrete $H$-reduced forward configurations is the bundle product Forw $_{d}^{H}=$ UForw $_{d}^{H} \times \Delta^{*} \operatorname{End}^{1} P_{d}$, where

$$
\operatorname{UForw}_{v}^{H}=H \operatorname{Str}_{v} \times \prod_{\emptyset \neq S \subsetneq[n]} \operatorname{End}^{1} P_{\alpha(v, S)}
$$

(in the underdetermined case, the component with $S=[n]$ and consequently $\operatorname{End}^{1} P_{\alpha}$ for the edge $\alpha=(v, v+$ $(1, \ldots, 1))$ are excluded).

The bundle UForw ${ }^{H} \rightarrow V$ shall be called bundle of underdetermined $H$-reduced forward configurations, and $\Delta^{*}$ End $^{1} P_{d} \rightarrow V$ bundle of forward endomorphisms.

Coming back to our original question for any admissible $H$-reduced field $(q, \psi)$ :

- Assume we know $q_{w}, \psi_{v_{0} w}$ for each $w$ in the region $k_{1}+\ldots+k_{n}<c+n$

- We want to determine $q_{u}, \psi_{v_{0} u}$ for each $u$ in the region $k_{1}+\ldots+k_{n}=c+n$

Take $v=u-(1, \ldots, 1)$, so that $\Delta(v)=(v, u)$. First observe that our assumption implies the knowledge of $u f_{v} \in \operatorname{UForw}_{v}^{H}$ and also $b k_{v} \in \operatorname{Back}_{v}^{H}$ associated to $(q, \psi)$.

Observe now that discrete Euler-Poincaré equations at $v$ can be written as:

$$
\operatorname{Leg}\left(u f_{v}, \psi_{\Delta(v)}\right)=\operatorname{Mom}\left(b k_{v}\right)
$$

where $u f \in \Gamma\left(\mathrm{UForw}^{H}\right), b k \in \Gamma\left(\operatorname{Back}^{H}\right), \psi_{\Delta} \in \Gamma\left(\Delta^{*} \operatorname{End}^{1} P_{d}\right)$ are the sections naturally associated to $(q, \psi)$.

\section{Definition 6.3}

We call integrator for the Legendre transformation Leg: UForw ${ }_{d}^{H} \times \Delta^{*} \operatorname{End}^{1} P_{d} \rightarrow \operatorname{Ad}^{*} P_{d}$, any mapping

$$
\Phi: \operatorname{UForw}_{d}^{H} \times \operatorname{Ad}^{*} P_{d} \rightarrow \Delta^{*} \text { End }^{1} P_{d}
$$

such that, for any $u f_{v} \in \mathrm{UForw}_{d}^{H}, \theta_{v} \in \operatorname{Ad}^{*} P_{v}$ there holds,

$$
\operatorname{Leg}\left(u f_{v}, \Phi\left(u f_{v}, \theta_{v}\right)\right)=\theta_{v}
$$

We say the integrator is a strong integrator if, moreover, $\operatorname{Leg}\left(u f_{v}, \cdot\right): \Delta^{*} \operatorname{End}^{1} P_{d} \rightarrow \operatorname{Ad}^{*} P_{d}$ is injective (in this case the integrator is unique).

We should observe, with the help of the implicit function theorem, that a necessary condition (and locally sufficient) for the existence of such a mapping is that the following linear mapping is invertible:

$$
\frac{\partial \operatorname{Leg}_{v}}{\partial \psi_{\Delta(v)}}\left(u f_{v}, \psi_{\Delta(v)}\right): V_{\psi_{\Delta(v)}} \operatorname{End}^{1} P_{\Delta(v)} \rightarrow \operatorname{Ad}^{*} P_{v}
$$

We may also use the source trivialisation to give an identification $V_{\psi_{\Delta(v)}} \operatorname{End}^{1} P_{\Delta(v)} \simeq \operatorname{Ad} P_{v}$, so that $\frac{\partial \operatorname{Leg}_{v}}{\partial \psi_{\Delta(v)}}\left(u f_{v}, \psi_{\Delta(v)}\right)$ represents a metric on $\operatorname{Ad} P_{v}$.

With the existence of such an integrator, we recover our main theorem: 
Theorem 6.1

Let $\Phi$ be an integrator for the Legendre transformation. Consider a locally defined admissible $H$-reduced field $(q, \psi) \in \Gamma\left(B_{c}, H \operatorname{Str}_{d}\right) \times \Gamma\left(B_{c}^{1}\right.$, End $\left.{ }^{1} P_{d}\right)$, defined on the initial condition band $B_{c}=\left\{v=\left(k_{1}, \ldots, k_{n}\right) \in\right.$ $\left.\mathbb{Z}^{n}: c-n \leq k_{1}+\ldots+k_{n} \leq c+n-1\right\}$. Consider $u f_{v} \in \mathrm{UForw}_{v}^{H}, b k_{v} \in$ Back $_{v}^{H}$ determined by this field at each $v \in S_{c}=\left\{v=\left(k_{1}, \ldots, k_{n}\right) \in \mathbb{Z}^{n}: k_{1}+\ldots+k_{n}=c\right\}$.

For $u \in S_{c+n}$ and $v=u-(1, \ldots, 1)$, the values

$$
\psi_{v u}=\Phi_{v}\left(u f_{v}, \mu_{v}\right), \quad q_{u}=q_{v} \psi_{v u}, \quad q_{v_{0} u}=\psi_{v v_{0}}^{-1} \psi_{v u}, \quad \text { with } \mu_{v}=\operatorname{Mom}_{v}\left(b k_{v}\right)
$$

extend $(q, \psi)$ to an admissible $H$-reduced field $(\bar{q}, \bar{\psi}) \in \Gamma\left(\Delta B_{c}, H \operatorname{Str}_{d}\right) \times \Gamma\left(\Delta B_{c}^{1}\right.$, End $\left.^{1} P_{d}\right)$ on the extended band $\Delta B_{c}=B_{c} \cup S_{c+n}$, in such a way that Euler-Poincaré equations (9) are satisfied at each vertex $v \in S_{c}$.

Moreover, if $\Phi$ is a strong integrator and $(q, \psi)$ is a critical admissible $H$-reduced field, taking the indicated values on the initial condition band $B_{c}$, then its values $q_{u}, \psi_{v_{0} u}$ for $u \in S_{c+n}$ are described by (11).

Proof

As the initial data is supposed to be admissible, at any 2-simplex contained in $B_{c}$ we have the discrete flatness condition and at each edge contained in $B_{c}$ we have the discrete parallelism condition for the $H$-structure $q$ with respect to $\psi$, as described in Definition 5.1.

In order that $q_{u}, \psi_{v_{0} u}$, for $u \in S_{c+n}$, determine an extension where Euler-Poincaré equations are satisfied at $v=u-(1, \ldots, 1)$, the elements $\psi_{v u}$ should verify:

$$
\operatorname{Leg}\left(u f_{v}, \psi_{v u}\right)=\operatorname{Mom}\left(b k_{v}\right)
$$

By Definition (10) of an integrator, the element $\psi_{v u}=\Phi_{v}\left(u f_{v}, \operatorname{Mom}_{v}\left(b k_{v}\right)\right)$ solves that equality. Moreover, if $\Phi$ is a strong integrator, $\operatorname{Leg}\left(u f_{v}, \cdot\right)$ is injective and the equation can not have another solution.

On the other hand, knowing $\psi_{v u}$, for $u \in S_{c+n}$ and $v=u-(1, \ldots, 1)$, allows to univocally determine $q_{u}=$ $q_{v} \psi_{v u}$ and $\psi_{v_{0} u}=\psi_{v v_{0}}^{-1} \psi_{v u}$, in order that the extended field remains admissible.

With these values there holds also $q_{v_{0}} \psi_{v_{0} u}=q_{v_{0}} \psi_{v v_{0}}^{-1} \psi_{v u}$, and using $q_{v_{0}} \psi_{v v_{0}}^{-1}=q_{v}$ (because the initial data was admissible, satisfying the parallelism condition), and then $q_{v} \psi_{v u}=q_{u}$ shows that $\left(q_{v_{0}}, q_{v}\right)$ is parallel for our choice $\psi_{v_{0} u}$.

Finally for a 2-simplex $\left(v_{0}, v_{1}, u\right)$ containing $u$ (which was not contained in the initial band) there holds $\psi_{v_{0} v_{1}} \psi_{v_{1} u}=\psi_{v_{0} v_{1}} \psi_{v v_{1}}^{-1} \psi_{v u}$ and using flatness on the 2-simplex $\left(v, v_{0}, v_{1}\right)$, (which holds because this simplex is contained in the initial band) we may use $\psi_{v_{0} v_{1}} \psi_{v v_{1}}^{-1}=\psi_{v v_{0}}^{-1}$ to conclude $\psi_{v_{0} v_{1}} \psi_{v_{1} u}=\psi_{v_{0} u}$, which is the flatness on the given 2-simplex.

We may illustrate the process in the 2-dimensional CFK simplicial complex in figure 2. It is shown how the initial condition band information is extended from the domain $k_{1}+k_{2} \leq c+n-1$ to $k_{1}+k_{2} \leq c+n$.

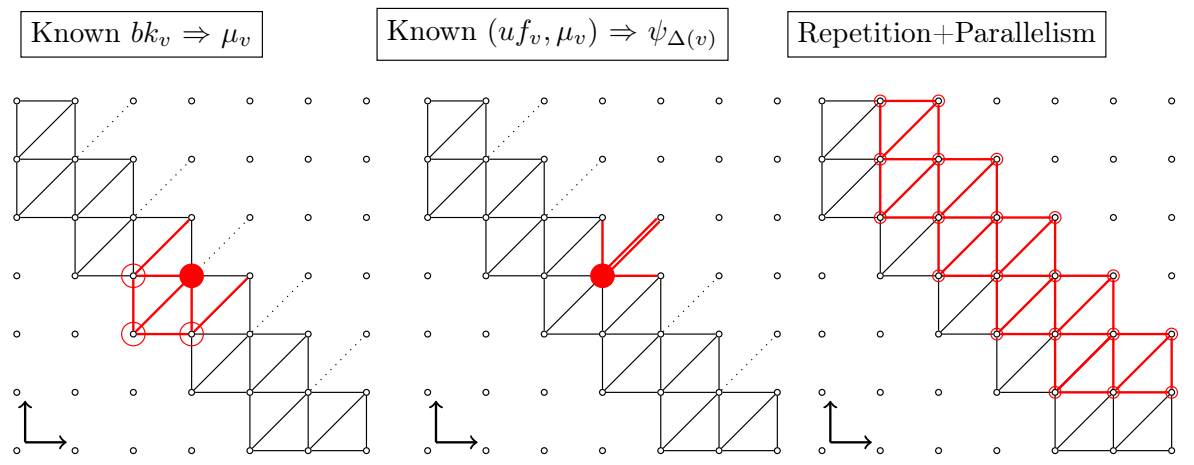

Figure 2. Algorithm of integration. 
This theorem indicates that, if there exists the integrator $\Phi$, we may obtain admissible discrete $H$-reduced fields $(q, \psi)$ satisfying Euler-Poincaré equations at vertices in the domain $k_{1}+\ldots+k_{n} \geq c$, using as initial data an admissible discrete $H$-reduced field defined only at vertices and edges included in the initial condition band $B_{c}$. The algorithm is:

For each vertex in the $c$-slice $v \in S_{c}$ :

1. Collect initial data to obtain backward configuration $b k_{v} \in \operatorname{Back}_{v}^{H}$.

2. Use momentum map to compute value $\mu_{v}=\operatorname{Mom}_{v}\left(b k_{v}\right) \in \operatorname{Ad}^{*} P_{v}$.

3. Collect initial data to obtain underdetermined forward configuration $u f_{v} \in \mathrm{UForw}_{v}^{H}$.

4. Use integrator to compute forward endomorphism $\psi_{v u}=\Phi\left(u f_{v}, \mu_{v}\right) \in$ End $^{1} P_{\Delta(v)}$.

5. Use parallelism condition to compute $q_{u}=q_{v} \psi_{v u}$ for the vertex $u=v+(1, \ldots, 1)$.

6. Use flatness condition to compute $\psi_{v_{0} u}=\psi_{v v_{0}}^{-1} \psi_{v u}$, for all edges with target $u$.

7. Collect all $H$-structures and fiber-to-fiber endomorphisms into new admissible discrete field $\left(q_{x}, \psi_{\bar{x}}\right)$ defined on $B_{c+1}$.

Iterate from the beginning for vertices in the $(c+1)$-slice.

All the fundamental computational cost lies in step 4, where the integrator may be given by some closed formula or may be approximated using some numerical algorithm that solves (10).

\section{Appendix}

\section{Appendix on quotient manifolds}

In this appendix we mention some results on quotients of manifolds, used in our work:

\section{Proposition 7.1}

[39, see Ch.3, Sec.12] Let $M$ be a smooth manifold and $R \subseteq M \times M$ an equivalence relation on $M$. The quotient space $M / R$ has a structure of smooth manifold for which $\pi: M \rightarrow M / R$ is a surjective submersion if, and only if, $R \subseteq M \times M$ is a closed submanifold, and the projection on the first component $\pi_{1}: R \rightarrow M$ is a smooth mapping. Moreover, the smooth structure on $M / R$ with this property is unique .

A well known consequence is

Corollary 7.1

Let $\lambda: G \rightarrow \operatorname{Aut}(M)$ be an action of a Lie group on a smooth manifold $M$. If $(g, m) \in G \times M \mapsto\left(\lambda_{g} m, m\right) \in$ $M \times M$ is smooth, injective and proper (smooth free proper action), the quotient space has a unique structure of smooth manifold for which $\pi: M \rightarrow M / G$ is a surjective submersion.

Finally, the quotient manifold structure has the property that we expect of a quotient, in the category of smooth manifolds:

\section{Proposition 7.2}

Let $\pi: M \rightarrow \bar{M}$ be a surjective submersion of smooth manifolds, and let $\bar{f}: \bar{M} \rightarrow N$ be a mapping from $\bar{M}$ to another smooth manifold $N$. For the mapping $f=\bar{f} \circ \pi$ there holds $f$ is smooth if, and only if, $\bar{f}$ is smooth; $f$ is a submersion if, and only if, $\bar{f}$ is a submersion, and $f$ is a surjective submersion if, and only if, $\bar{f}$ is a surjective submersion.

\section{Appendix on Polytopes}

Recall that for arbitrary points $\left(v_{0}, \ldots, v_{m}\right)$ of an affine space and any choice of weights $\lambda_{0}, \ldots, \lambda_{n}$ (whith the condition $\lambda_{0}+\lambda_{1}+\ldots+\lambda_{m}=1$ ) there exists a notion of affine combination $v=\lambda_{0} v_{0}+\lambda_{1} v_{1}+\ldots+\lambda_{m} v_{m}$. This affine combination is called a convex combination if all weights $\lambda_{i}$ are non-negative, and an open convex 
combination is all weights $\lambda_{i}$ are positive (observe that $v_{0}$ is an open convex combination of the set $\left\{v_{0}\right\}$, but it is not an open convex combination of $\left\{v_{0}, v_{1}\right\}$ ). The affine hull $\left\langle v_{0}, v_{1}, \ldots, v_{m}\right\rangle$ of a set of points is the set of its affine combinations. The convex hull $\left[v_{0}, \ldots, v_{m}\right]$ is the set of its convex combinations. The open convex hull $] v_{0} \ldots v_{m}[$ is the set of its open convex combinations. In particular the convex hull $[r, s]$ of two points $r, s$ is called the segment joining these points, and the open convex hull $] r, s[$ is called the open segment joining both points (observe that ]$r, s[\subseteq[r, s] \subseteq\langle r, s\rangle$, with equality only in the case $r=s)$. A subset $B \subset \mathbb{R}^{n}$ is called convex if for any pair of points $r, s \in B$, the associated open segment $] r, s[$ is also contained in $B$.

Convex polytopes on $\mathbb{R}^{n}$ are subsets $B \subseteq \mathbb{R}^{n}$ that can be described as the convex hull of a finite number of points in $\mathbb{R}^{n}$. We suggest $[3,43]$ as a reference for the needed theory on convex polytopes. In particular, we shall use the notion of face of a polytope.

Definition 7.1

A face of a closed convex set $B \subseteq \mathbb{R}^{n}$ is any nonempty convex subset $\emptyset \neq A \subseteq B$ such that, for any pair of elements $r, s \in B$ with $r \notin A$, the open segment $] r, s[$ determined by these points does not intersect $A$.

$$
A \text { convex, } \quad \emptyset \neq A \subseteq B, \quad(r \notin A \Rightarrow] r, s[\cap A=\emptyset) \forall r, s \in B
$$

Observe that with this definition $B$ is a face of itself, but $\emptyset$ is not a face.

A point $v$ is said to be an extreme point of the closed convex set $B$, if $A=\{v\}$ is a face of $B$ (that is, $v \in B$ but it is not contained in any open segment determined by elements in $B$ ). Extreme points of a polytope $B$ is a finite set $\operatorname{Ext}(B)$, and the convex hull of these extreme points is again $B$ itself.

We denote $A \prec B$ to indicate that $A$ is a face of $B$. This establishes an order on the set of polytopes (for transitivity, consult [3]).

We recall now the fact that any polytope can be characterized as the convex hull of its extreme points. Moreover, none of the extreme points can be written as a convex combination of the remaining extreme points. Conversely, given any finite set of points where none of them is a convex combination of the remaining ones, the polytope generated by these points has as extreme points the given finite set.

\section{Proposition 7.3}

The computation of convex hull and the computation of extreme points establishes a one-to-one correspondence between convex polytopes in $\mathbb{R}^{n}$ and subsets $\left\{v_{0}, v_{1}, \ldots, v_{m}\right\} \subseteq \mathbb{R}^{n}$ of points, where none of them is a convex combination of the remaining ones.

Proof

There are certain characteristics that relate polytopes with its extremal points [43]:

- For each polytope $B$ holds $[\operatorname{Ext}(B)]=B$.

- $B=\left[v_{0}, v_{1}, \ldots, v_{n}\right] \Rightarrow \operatorname{Ext}(B) \subseteq\left\{v_{0}, v_{1}, \ldots, v_{n}\right\}$.

- $v_{0} \in\left[v_{1}, \ldots, v_{n}\right] \Rightarrow\left[v_{0}, v_{1}, \ldots, v_{n}\right]=\left[v_{1}, \ldots, v_{n}\right]$.

Therefore, when we have a polytope generated as a convex hull of $v_{0}, \ldots, v_{n}$, we may firstly eliminate vertices that are in the convex hull of the remaining ones and still get generators for the same polytope. Assume now that $v_{0}, \ldots, v_{n}$ are such vertices, where none of them is a convex combination of the remaining ones and with $B=\left[v_{0}, \ldots, v_{n}\right]$. In this case, we get $v_{i} \in B=[\operatorname{Ext}(B)]$, hence $v_{i}$ is a convex combination of the extreme points of $B$. In the case that $v_{i}$ is not itself an extreme point, from $\operatorname{ext}(B) \subset\left\{v_{0}, \ldots, v_{i-1}, v_{i+1}, \ldots, v_{n}\right\}$ we would conclude that $v_{i}$ would be a convex combination of the remaining elements, in contradiction with our assumption.

This leads to the following:

\section{Definition 7.2}

We call abstract convex polytope on $\mathbb{R}^{n}$, any finite set of points $\beta=\left\{v_{0}, v_{1}, \ldots, v_{m}\right\} \subset \mathbb{R}^{n}$, such that none of them is a convex combination of the remaining ones. The convex hull, $[\beta] \subset \mathbb{R}^{n}$, of this set of points is called the convex polytope associated to $\beta$. 
If these points are affinely independent points (none of them is an affine combination of the remaining ones), the abstract convex polytope is called an abstract simplex and the polytope is called a simplex.

We call dimension of an abstract polytope the dimension of the affine space generated by this set of points. For a simplex with $m+1$ points, its dimension is $m$. Observe that all 1-dimensional polytopes are in fact segments $[r, s]$ determined by two extreme points and therefore are simplices.

We say an abstract polytope $\alpha$ is a face of another abstract polytope $\beta$, if the associated convex hull $\bar{\alpha}$ is a face of $\bar{\beta}$. This determines an order relation $\alpha \prec \beta$ in the set of abstract polytopes.

In the case of an $m$-dimensional simplex generated by $\left\{v_{0}, \ldots, v_{m}\right\}$, the convex polytopes generated by any subset are faces of the simplex. In the case of an arbitrary polytope generated by $\left\{v_{0}, \ldots, v_{m}\right\}$, a given subset of points generates a smaller polytope, but not necessarily a face.

\section{Acknowledgements}

This work was partially supported by Fundação para a Ciência e a Tecnologia (Portuguese Foundation for Science and Technology) through the project UID/MAT/00297/2013 (Centro de Matemática e Aplicações) and UID/MAT/04561/2013 (Centro de Matemática, Aplicações Fundamentais e Investigação Operacional of Universidade de Lisboa CMAF-CIO).

\section{References}

1. D.N. Arnold, R.S. Falk, R. Winther. Finite element exterior calculus, homological techniques, and applications. Acta Numerica 15 (2006), 1-155.

2. A.I. Bobenko, Yu.B. Suris. Discrete Lagrangian reduction, discrete Euler-Poincaré equations, and semidirect products. Letters in Mathematical Physics 49.1 (1999): 79-93.

3. A. Brondsted, An introduction to convex polytopes. Vol. 90. Springer Science \& Business Media, 2012.

4. A.C. Casimiro, C. Rodrigo, First variation formula and conservation laws in several independent discrete variables. J. Geom. Phys. 62.1 (2012), 61-86

5. A.C. Casimiro, C. Rodrigo, First variation formula for discrete variational problems in two independent variables. RACSAM Rev. R. Acad. A 106.1 (2012), 111-135.

6. A.C. Casimiro, C. Rodrigo, Reduction of Forward Difference Operators in Principal G-bundles. Statistics Opt. Inform. Comput., Vol. 6, (2018), 42-85.

7. M. Castrillón López, Field theories: reduction, constraints and variational integrators. Revista de la Real Academia de Ciencias Exactas, Físicas y Naturales. Serie A. Matematicas 106(1) (2012): 67-74

8. M. Castrillón López, P. Chacón, P.L. García, Lagrange-Poincaré reduction in affine principal bundles, J.Geom.Mech., 5 (vol 4), $399-414$

9. M. Castrillón López, P.L. García, T.S. Ratiu, Euler-Poincaré reduction on principal bundles, Lett. Math. Phys. 58 (2001) 167-180

10. M. Castrillón López, P.L. García, C. Rodrigo, Euler-Poincaré reduction in principal bundles by a subgroup of the structure group, Journal of Geometry and Physics, Volume 74, December 2013, Pages 352-369

11. S. H. Christiansen, H. Z. Munthe-Kaas, B. Owren, Topics in structure-preserving discretization. Acta Numerica 20 (2011), 1-119.

12. J. Cortés, S. Martínez, Non-holonomic integrators. Nonlinearity 14 (2001) 1365-1392.

13. K. Crane, Discrete connections for geometry processing. Diss. California Institute of Technology, 2010.

14. F. Demoures, F. Gay-Balmaz, S. Leyendecker, S. Ober-Bl?baum, T.S. Ratiu, T. S., Y. Weinand. Discrete variational Lie group formulation of geometrically exact beam dynamics. Numerische Mathematik, 130(1), (2015) 73-123.

15. F. Demoures, F. Gay-Balmaz, T.S. Ratiu. Multisymplectic variational integrators and space/time symplecticity. Analysis and Applications, 14(03), (2016) 341-391.

16. D.C.P. Ellis, F. Gay-Balmaz, D.D. Holm, T.S. Ratiu, Lagrange-Poincaré field equations, J. Geom. Phys. 61 (11) (2011) $2120-2146$.

17. J. Fernandez, M. Zuccalli. A geometric approach to discrete connections on principal bundles. Journal of Geometric Mechanics . Dec2013, Vol. 5 Issue 4, p433-444. 12p.

18. A. Fernández, P.L. García, C. Rodrigo. Stress-energy-momentum tensors for natural constrained variational problems. Journal of Geometry and Physics 49.1 (2004): 1-20.

19. A. Fernández, P. García, C. Rodrigo, Variational integrators in discrete vakonomic mechanics. Rev. R. Acad. A 106 (2012) $137-159$.

20. E.S. Gawlik, P. Mullen, D. Pavlov, J.E. Marsden, M. Desbrun. Geometric, variational discretization of continuum theories. Physica D: Nonlinear Phenomena, 240(21), (2011) 1724-1760.

21. H.-Y. Guo, K. Wu, On variations in discrete mechanics and field theory, J. Math. Phys 44(12) (2003) 5978-6004.

22. E. Hairer, C. Lubich, G. Wanner, Geometric numerical Integration: Structure-Preserving Algorithms for Ordinary Differential Equations. Springer Series in Computational Mathematics 31. Springer Verlag Berlin Heidelberg, New York, 2004.

23. R. Hermann Gauge Fields and Cartan-Ehresmann Connections, Part A. Vol. 10. Math Science Press, 1975.

24. A. Iserles, H.Z. Munthe-Kaas, S. P. N?rsett, A. Zanna. Lie-group methods. Acta Numerica, (2005) 1-148. 
25. M. B. Kobilarov, J. E. Marsden. Discrete geometric optimal control on Lie groups IEEE Transactions on Robotics 27.4 (2011): 641-655.

26. M. Kobilarov. Solvability of Geometric Integrators for Multi-body Systems. Multibody Dynamics. Springer International Publishing, 2014. 145-174.

27. R. McLachlan, M. Perlmutter. Integrators for nonholonomic mechanical systems Journal of nonlinear science 16.4 (2006): $283-328$.

28. R.I. Mc Lachlan, G.R.W. Quispel, Geometric integrators for ODEs. J. Phys. A: Math. Gen. 39 (2006), 5251-5285.

29. M. Leok, J.E. Marsden, and A.D. Weinstein. A discrete theory of connections on principal bundles. arXiv preprint math/0508338 (2005).

30. M. de León, D. Martín de Diego, A. Santamaría Merino, Geometric integrators and nonholonomic mechanics. J. Math. Phys. 45(3) (2004) 1042-1064.

31. M. de León, J.C. Marrero, D. Martín de Diego, Some applications of semi-discrete variational integrators to classical field theories. Qual. Th. Dyn. Syst. 7.1 (2008), 195-212.

32. A. Lew, J.E. Marsden, M. Ortiz, M. West, Asynchronous variational integrators. Arch. Rat. Mech. Anal. 167 (2003), 85-146.

33. J.E. Marsden, G.W.Patrick, and S. Shkoller. Multisymplectic geometry, variational integrators, and nonlinear PDEs. Commun. Math. Phys. 199.2 (1998), 351-395.

34. J.E. Marsden, S. Pekarsky, S. Shkoller. Symmetry reduction of discrete Lagrangian mechanics on Lie groups. Journal of geometry and physics 36.1 (2000): 140-151.

35. J. E. Marsden, M. West, Discrete mechanics and variational integrators. Acta Numer. 10 (2001) 357-514.

36. S. P. Novikov, Discrete connections and linear difference equations, Tr. Mat. Inst. Steklova 247 (2004), 18601, math-ph/0303035

37. D. W. Moore, Simplical mesh generation with applications. PhD Thesis Cornell University, 1992.

38. A.N. Sengupta, Connections over two-dimensional cell complexes. Rev. Math. Phys. 16.3 (2004), 331-352.

39. J.P. Serre, Lie algebras and Lie groups, Lectures given at Harvard University, 1964, Benjamin, New York, 1965.

40. J. Vankerschaver. Euler-Poincaré reduction for discrete field theories. Journal of mathematical physics, 48(3), (2007) 032902.

41. J. Vankerschaver, F. Cantrijn. Discrete Lagrangian field theories on Lie groupoids. Journal of Geometry and Physics 57(2) (2007): $665-689$.

42. J.M. Wendlandt, J.E. Marsden, Mechanical integrators derived from a discrete variational principle. Physica D 106 (1997), $223-246$.

43. G.M. Ziegler, Lectures on polytopes. Vol. 152. Springer Science and Business Media, 2012. 